Transformation, denn aus

$$
\sum\left(\dot{q}_{i} \mathrm{~d} p_{i}-\dot{p}_{i} \mathrm{~d} q_{i}\right)=\mathrm{d}_{t} H\left(q_{i}, p_{i}, t\right)
$$

folgen die Hamiltonschen gewöhnlichen Differentialgleichungen

$$
\dot{q}_{i}=\frac{\partial H}{\partial p_{i}}, \quad \dot{p}_{i}=-\frac{\partial H}{\partial q_{i}} \quad(i=1, \ldots, f),
$$

die den Übergang des Systems von der Zeit $t$ zur Zeit $t+\mathrm{d} t$ beschreiben.

Nun sei umgekehrt eine Hamilton-Funktion $H(q, p, t)$ und mit ihr vermöge (15) eine infinitesimale kanonische Transformation gegeben. Es entsteht die Frage nach einer kanonischen Transformation, welche die Zustände des Systems für jede Zeit mit den Zuständen zur Zeit 0 verbindet; anders ausgedrückt, es ist eine Wirkungsfunktion $S(\alpha, q, t)$ gesucht, welche nach (10) oder

$$
p_{i}=\frac{\partial S}{\partial q_{i}}, \quad \beta_{i}=-\frac{\partial S}{\partial \alpha_{i}} \quad(i=1, \ldots, f)
$$

diese Transformation erzeugt.
Für sie bestehen die Beziehungen:

$$
\frac{\mathrm{d}}{\mathrm{d} t} S\left(a_{i}, q_{i}, t\right)=\sum \frac{\partial S}{\partial q_{i}} \dot{q}_{i}+\frac{\partial S}{\partial t}
$$

und nach (9), (14) und (16)

$$
\begin{aligned}
& \frac{\mathrm{d}}{\mathrm{d} t} S\left(\alpha_{i}, q_{j}, t\right)=\dot{T}\left(\alpha_{i}, \beta_{i}, t\right) \\
& =\sum p_{i} \dot{q}_{i}-H\left(q_{i}, p_{i}, t\right)=\sum \frac{\partial S}{\partial q_{i}} \dot{q}_{i}-H\left(q_{j}, \frac{\partial S}{\partial q_{j}}, t\right) .
\end{aligned}
$$

Die Wirkungsfunktion $S\left(\alpha_{i}, q_{i}, t\right)$ muß also der Hamilton-Jacobischen partiellen Differentialgleichung

$$
\frac{\partial S}{\partial t}+H\left(q_{i}, \frac{\partial S}{\partial q_{i}}, t\right)=0
$$

genügen, und umgekehrt beschreibt jedes vollständige Integral von (17) vermöge (16) das mechanische System.

Herrn Prof. E. H ü c kel danke ich für die anregenden Diskussionen, aus denen diese Darstellung hervorgegangen ist.

\title{
Zur Thermodynamik der irreversiblen Prozesse $\mathrm{I}^{*}$
}

\author{
Von Rolf HaAse \\ Aus dem Physikalisch-Chemischen Institut der Universität Marburg (Lahn) \\ (Z. Naturforschg. 6 a, 420-437 [1951]; eingegangen am 15. Mai 1951)
}

Die Gleichungen für Enćropieströmung und Entropieerzeugung sowie die phänomenologischen Ansätze mit den Onsa gerschen Reziprozitätsbeziehungen werden im Anschluß an Meixner und Prigogine für folgende Fälle aufgestellt und diskutiert: beliebig viele chemische Reaktionen in offenen homogenen Systemen, Kopplung von Materie- und Wärmeübergang zwischen zwei Phasen eines polythermen Systems, beliebige irreversible Prozesse in kontinuierlichen Systemen, die aus isotropen Medien bestehen. Dabei wird in allen Fällen eine beliebige Anzahl von Stoffen vorausgesetzt. Allgemeine Ungleichungen für die phänomenologischen Koeffizienten werden formuliert. Ferner wird folgendes gezeigt: Bei polythermen bzw. kontinuierlichen Systemen kann nach einer bestimmten Vorschrift, die durch Betrachtungen an offenen homogenen Systemen nahegelegt wird, aus dem „Wärmestrom“ ein Anteil abgespalten werden, der sich auf den Wärmetransport durch die Materieströme bzw. Diffusions. ströme bezieht. Der so gebildete „reduzierte Wärmestrom“ ermöglicht eine besonders einfache Formulierung der Resultate der thermodynamisch-phänomenologischen Theorie. Insbesondere gelten für die mit dem „reduzierten Wärmestrom“ definierten beiden „Wärmeleitfähigkeiten“ $\lambda$ (für homogene Durchmischung) und $\varkappa$ (für stationären Zustand) folgende Aussagen:

$$
\lambda>0, \varkappa>0, \lambda-\varkappa=\frac{1}{T} \sum B_{i} Q_{i}>0,
$$

worin die $B_{i}$ clie phänomenologischen Koeffizienten für die „Überlagerungsprczesse“ (Thermoosmose, Thermodiffusion, thermoelektrische Erscheinungen usw.) und die $Q_{i}$ die „Überführungswärmen “ sind, die sich wiederum durch die $B_{i}$ und die phänomenologischen Koeffizienten für den isothermen Massentransport zwischen verschiedenen Phasen bzw. für die isotherme Diffusion ausdrücken lassen. Da speziell die erste Ungleichung bei Wahl eines anderen „Wärmestromes“ nicht erfüllt zu sein braucht, folgt hieraus eine gewisse Vorzugsstellung des „reduzierten Wärmestromes“.

* Abgeänderter Auszug aus: Habil.-Schrift Marburg 1950. 
$\mathrm{D}$ ie thermodynamisch-phänomenologische Theorie der irreversiblen Prozesse ist in ihrer modernen Form hauptsächlich von de D ond e ${ }^{1}$, Onsager ${ }^{2}$, Eckart ${ }^{3}$, Meixner ${ }^{4}, P_{\text {rigogine }}{ }^{5}$ und deGroot ${ }^{6}$ entwickelt worden. Das Ziel dieser Theorie sind gewisse allgemeine Aussagen über die gegenseitige Überlagerung von verschiedenen irreversiblen Vorgängen. Die Methodik besteht in einer erweiterten Anwendung der Begriffe und Gesetze der Thermodynamik.

Die systematisch durchgeführte Theorie beginnt mit der sog. "Gibbsschen Fundamentalgleichung“, deren Gültigkeit - in Erweiterung ihrer ursprünglichen Verwendung in der klassischen Thermodynamik - unter gewissen Voraussetzungen für irreversible Änderungen postuliert wird. Aus dieser Gleichung ergibt sich - bei Berücksichtigung der Erhaltungssätze der Physik - die „Entropiebilanzgleichung“, d.h. eine Beziehung für die zeitliche Änderung der Entropie des Systems bzw. des Volumenelementes. Denjenigen Anteil der Änderungsgeschwindigkeit der Entropie des betreffenden Systems bzw. der Entropiedichte im betrachteten Volumenelement, der verursacht wird durch Wärme- und Materieaustausch mit der Umgebung (durch die Begrenzungsflächen des Systems oder des Volumenelementes hindurch), nennt man „Entropieströmung“ bzw. „Quelldichte des Entropiestromes“, während man den restlichen Teil, der durch die im Inneren des Systems bzw. des Volumenelementes ablaufenden irreversiblen Vorgänge bedingt ist, als „Entropieerzeugung“ $\Theta$ bzw. „lokale Entropieerzeugung“ $\vartheta$ bezeichnet. Die „Dissipationsfunktion“, d.h. $\operatorname{der}$ Ausdruck $T \Theta$ bzw. $T \vartheta$, besteht aus einer Summe von zweigliedrigen Produkten der Form $\sum_{\gamma} X_{\gamma} L_{\gamma}$, wobei die $X_{\gamma}$ generalisierte "Ströme“ und die $L_{\gamma}$ generalisierte „Kräfte“ sind, die im Gleichgewicht alle einzeln verschwinden $(\Theta=0$ bzw. $\vartheta=0$ ).

Der Zweite Hauptsatz der Thermodynamik wird nun zu der Aussage erweitert, daß für jeden Zeitpunkt bei Ablauf irreversibler Prozesse gilt: $\Theta>0$ bzw. $\vartheta>0$.

Aber erst der nächste Schritt, die Verwendung der

1 Th. de Donder, L'Affinité, Paris 1927-1936.

2 L. O n s a g e r, Physic. Rev. 37, 405 [1931]; 38, 2265

[1931]; Ann. New York Acad. Sci. 46, 241 [1945].

3 C. E ck a rt, Physic. Rev. 58, 267, 269 [1940].

4 J. M e ixner, Ann. Physik (5) 35, 701 [1939]; 36, 578 [1939]; 39, 333 [1941]; 41, 409 [1942]; 43, 244 [1943]; Z. physik. Chem. Abt. B 53, 235 [1943]. Z. Naturforschg. 4 a, 594 [1949]. „phänomenologischen Ansätze“, führt zu konkreten Aussagen über bestimmte irreversible Prozesse. Diese Ansätze stellen homogene lineare Beziehungen zwischen „Strömen“ und „Kräften“ dar. Für die hierin auftretenden „phänomenologischen Koeffizienten“ gilt ein wichtiges allgemeines Theorem, das aus dem „Prinzip der mikroskopischen Reversibilität" folgt: der Reziprozitätssatz von O n s a g e r ${ }^{2,4,6}$. Die „Reziprozitätsbeziehungen “ fordern Symmetrie der Matrix der phänomenologischen Koeffizienten und können als Theorem der makroskopischen Physik neben die Hauptsätze der Thermodynamik gestellt werden. Sie folgen aber keineswegs aus Betrachtungen über makroskopische Symmetrieeigenschaften, sondern sind - wie angedeutet - generell im Rahmen der Molekulardynamik zu begründen.

Die Zuordnung thermodynamischer Begriffe (Temperatur, Entropie usw.) zu einem System bzw. einem Systemteil, in dem sich irreversible Prozesse abspielen, und insbesondere die Verwendung der G i b b s schen Fundamentalgleichung bedarf einer Rechtfertigung [Meixner ${ }^{4}, \mathrm{~T}$ olm an und $\mathrm{Fine}^{7}$, Bridg$\operatorname{man}^{8}$, Callen ${ }^{9}$, Prigogine ${ }^{10}$.

Es zeigt sich, daß bei chemischen Reaktionen „hinreichend langsamer Verlauf" und bei den übrigen irreversiblen Vorgängen "Gleichgewichtsnähe“ vorausgesetzt werden muß. Die nähere Diskussion der Bedeutung dieser Voraussetzungen ist nur für spezielle kinetische Modelle durchführbar. Trotzdem ist folgendes von vornherein wahrscheinlich und wird durch den Erfolg nachträglich zur Gewißheit: Im Bereich der linearen "phänomenologischen Ansätze“ sind die Bedingungen für die Anwendbarkeit der Theorie praktisch erfüllt. Diese Ansätze stellen in bezug auf die chemischen Reaktionen sogar eine weitergehende Einschränkung als die Voraussetzung der Gültigkeit der Gibbsschen Fundamentalgleichung dar: sie fordern nicht nur langsamen Verlauf, sondern auch Gleichgewichtsnähe. Die phänomenologischen Ansätze enthalten aber außerdem alle linearen Gesetze für irreversible physikalische Prozesse, z. B. O h m sches, F o u r i e r sches und F i c k sches Gesetz,

5 I. Prig ogine, Etude thermodynamique des Phénomènes irréversibles. Paris u. Lüttich 1947.

${ }_{6}$ S. R. de G r o o t, L'effet Soret, Diss. Amsterdam 1945; J. Physique Radium 8, 188, 193 [1947]; Physica 13, 555 [1947].

7 R. C. Tolman u. P. C. F in e, Rev. mod. Physics 20, 51 [1948].

8 P.W. B ri d g m a n, Rev. mod. Physics 22, 56 [1950].

${ }^{9}$ H. B. C a ll e n, Physic. Rev. 73, 1349 [1948].

10 I. Prigogine, Physica 15, 272 [1949]. 
und deren sinngemäße Verallgemeinerungen für die Überlagerungsprozesse (thermoelektrische Erscheinungen, Thermoosmose, Thermodiffusion usw.). Die Vorzüge unserer Theorie beruhen daher auf der Möglichkeit allgemeingültiger, von speziellen Modellen unabhängiger Aussagen, die insbesondere für beliebige Aggregatzustände und beliebige Zahl von Stoffen gültig sind.

In früheren Arbeiten 11,12, 13 wurde am Beispiel der Thermodiffusion in binären Systemen ausführlich dargelegt, wie die Ons a ger schen Reziprozitätsbeziehungen und der positiv definite Charakter der lokalen Entropieerzeugung zu einer Reihe genereller Aussagen über meßbare Koeffizienten bei Transporterscheinungen führen.

$\mathrm{M}$ e i x n e $\mathrm{r}^{4}$ hat darauf hingewiesen, daß - innerhalb genau angebbarer Grenzen - die Wahl der „Ströme“ und „Kräfte“ für die Aufstellung der linearen phänomenologischen Ansätze willkürlich ist. Deshalb unterscheiden sich die von verschiedenen Autoren gegebenen Darstellungen beträchtlich, wenn auch die endgültigen Aussagen der Theorie inhaltsgleich sind.

Bei Durchsicht der Rechnungen der einzelnen Autoren gelangt man zu dem Schluß, daß die wesentlichen Unterschiede und damit die formalen Schwierigkeiten beim Vergleich der verschiedenen Rechnungen in erster Linie durch die Verschiedenheit der Wahl des „Wärmestromes“ bedingt sind. Bereits im speziellen Falle der Thermodiffusion in binären Gemischen ${ }^{12}$ fiel dem Verfasser auf, daß bei Zugrundelegung eines schon von $\mathrm{Prigog}$ in $\mathrm{e}^{5}$ benutzten „Wärmestromes“ die Rechnungen besonders einfach werden und die beiden hierdurch definierten „Wärmeleitfähigkeiten“ stets positiv sind. In der vorliegenden Arbeit wird gezeigt, wie die Wahl eines „reduzierten Wärmestromes“ bei beliebig komplizierten Prozessen zu Vereinfachungen der Theorie im angedeuteten Sinne führt.

11 R. H a a s e, Z. Physik 127, 1 [1950].

12 R. H a a s e, Z. Elektrochem. angew. physik. Chem. 54, 450 [1950].

13 R. H a a e, Z. physik. Chem. 196, 219 [1950].

14 Von diesen allein handelt die de Dondersche Thermodynamik 1 .

15 Damit diese Vorgänge in uniformen Systemen möglich sind, muß, streng genommen, gefordert werden, daß die Geschwindigkeit des Ausgleichs von Temperatur-, Druck- und Konzentrationsunterschieden, die sich im System durch chemische Reaktionen und Stoffübergänge zwischen den Phasen sowie evt. Massenaustausch mit der Umgebung ausbilden, groß ist im Vergleich zu den Reaktions- und Materieübertrittsgeschwindigkeiten.
Da später weitere allgemeine Betrachtungen durchgeführt werden sollen, stellen wir hier die generellen Züge der Theorie in knapper, aber zusammenhängender Form dar.

\section{Allgemeines}

Wir betrachten zunächst ein System, das entweder homogen ist oder aus mehreren homogenen Teilen („Phasen“) zusammengesetzt ist; es bestehe sowohl mit der Umgebung als auch zwischen den einzelnen Phasen thermisches und (soweit keine semipermeablen Wände vorhanden) mechanisches Gleichgewicht, ohne daß die Bedingungen des chemischen und heterogenen stofflichen Gleichgewichts erfüllt zu sein brauchen. Ein solches System heißt ,uniform“ " . Irreversible Vorgänge, die in einem System ablaufen, das zu jedem Zeitpunkt als „uniform“ vorausgesetzt werden darf, nennen wir kurz,,irreversible Prozesse in uniformen Systemen "14. Schließen wir äußere Kraftfelder, makroskopische Bewegungen usw. aus, so bestehen solche Prozesse in chemischen Reaktionen und Stoffübergängen zwischen Phasen gleicher Temperatur und (bei Fehlen semipermeabler Wände) gleichen Druckes ${ }^{15}$.

Ein System, das aus mehreren homogenen Teilen („Phasen“) verschiedener Temperatur (und eventuell verschiedenen Druckes) besteht, bezeichnen wir als „polythermes System“ ${ }^{5}$. Bei irreversiblen Vorgängen in polythermen Systemen ist außer mit chemischen Reaktionen und Stoffübergängen zwischen den Phasen auch mit einem Wärmetransport zwischen den verschieden temperierten Teilen des Systems zu rechnen ${ }^{16}$.

Schließlich ist es möglich, daß in einem System Temperatur, Druck, Konzentrationen usw. kontinuierlich von Punkt zu Punkt variieren, so daß von einer endlichen Zahl von Phasen nicht mehr die Rede sein kann. Dann haben wir es mit einem „kontinuierlichen System “ zu tun ${ }^{5}$. Bei irreversiblen Erscheinungen in kontinuierlichen Systemen sind Materietransport mit innerer Reibung, Diffusion, Wärmeleitung usw. zu berücksichtigen.

Den kontinuierlichen Systemen können die uniformen und polythermen Systeme als ,diskontinuierliche Systeme“ gegenübergestellt werden.

Unter den schon angedeuteten Vorausetzungen

16 Damit diese Vorgänge in polythermen Systemen möglich sind, muß allgemein gefordert werden, daß Temperatur-, Druck- und Konzentrationsausgleich innerhalb einer Phase schnell erfolgen im Vergleich zu den chemischen Reaktionen und zum Energie- und Stofftransport zwischen den Phasen. 
- „langsamer Ablauf“ bei chemischen Reaktionen bzw. "Gleichgewichtsnähe“ bei anderen irreversiblen Prozessen - gilt die „Gibbssche Fundamentalgleichung " auch bei irreversiblen Änderungen für jede Phase eines diskontinuierlichen Systems bzw. für jedes Volumenelement eines kontinuierlichen Systems. Für die betrachtete Phase bzw. das Volumenelement sei $T$ die absolute Temperatur, $S$ die Entropie, $U$ die innere Energie, $p$ der Druck, $V$ das Volumen, $\mu_{i}$ das chemische Potential des Stoffes $i$, bezogen auf die Masseneinheit, $m_{i}$ die Masse des Stoffes $i$ und $N$ die Zahl der Stoffe. Dann lautet die Gibbssche Fundamentalgleichung:

$$
T \mathrm{~d} S=\mathrm{d} U+p \mathrm{~d} V-\sum_{i=1}^{N} \mu_{i} \mathrm{~d} m_{i} .
$$

Bezeichnen wir mit $M_{i}$ die Molmasse, mit $n_{i}$ die Zahl der Mole und mit $\bar{\mu}_{i}$ das molare chemische Potential des Stoffes $i$, so gilt:

$$
\begin{gathered}
\bar{\mu}_{i}=M_{i} \mu_{i}, \\
\sum \mu_{i} \mathrm{~d} m_{i}=\sum \bar{\mu}_{i} \mathrm{~d} n_{i} .
\end{gathered}
$$

Im Falle kontinuierlicher Systeme ist es zweckmäßig, Gl. (1) auf die Masseneinheit des Volumenelementes zu beziehen, d. h. $m_{1}+m_{2}+\ldots+m_{N}=1$ zu setzen.

Es sei $m$ die Gesamtmasse und

$$
x_{i} \equiv \frac{m_{i}}{\sum_{i=1}^{N} m_{i}}=\frac{m_{i}}{m}
$$

der „Massenbruch“ des Stoffes $i$. Ferner führen wir folgende Bezeichnungsweise ein: Wenn eine beliebige extensive Zustandsfunktion $Z$ genannt wird, so sei $\bar{Z} \equiv Z / m$ die spezifische Größe und

$$
Z_{i} \equiv\left(\partial Z / \partial m_{i}\right)_{T, p, m_{j}}(j \neq i)
$$

die partielle Größe (je Masseneinheit) des Stoffes $i$. Außer der gelegentlichen Verwendung des molaren chemischen Potentials $\left(\bar{\mu}_{i}\right)$ benutzen wir in der vorliegenden Arbeit die (mittleren) molaren und partiellen molaren Zustandsfunktionen nicht.

Wir können also an Stelle von Gl. (1) schreiben:

$$
T \mathrm{~d} \bar{S}=\mathrm{d} \bar{U}+p \mathrm{~d} \bar{V}-\sum_{i=1}^{N} \mu_{i} \mathrm{~d} x_{i} .
$$

Die Massen bzw. Molzahlen in einer Phase können sich sowohl durch innere Ursachen (Ablauf von homogenen chemischen Reaktionen) als auch durch Materieaustausch mit der Umgebung ändern. Deshalb ist folgende begriffliche Unterscheidung wichtig. Ein beliebiges thermodynamisches System, das mit der Umwelt sowohl Energie als auch Materie austauschen kann, heißt nach D ef a y ${ }^{17}$ ein „offenes System “, während ein solches, das mit der Umgebung zwar Energie, nicht aber Materie auszutauschen vermag, als "geschlossenes System“ bezeichnet wird. Mit diesem darf ein „abgeschlossenes System“, das sowohl stofflich als auch energetisch von der Außenwelt isoliert ist, nicht verwechselt werden.

Soweit wir hier des Zusammenhangs wegen bekannte Beziehungen ableiten müssen, folgen wir bei den „uniformen“ und ,polythermen“"Systemen hauptsächlich der Darstellung von Prig o g i n $\mathrm{e}^{5}$ und bei den „kontinuierlichen“ Systemen teils derjenigen von $\mathrm{Meixne{ } ^ { 4 }}$ und teils derjenigen von Prigogine.

\section{Uniforme Systeme}

a) Bilanzgleichungen

Wir betrachten den einfachsten Fall von irreversiblen Prozessen in uniformen Systemen: homogene chemische Reaktionen, d.h. chemische Umsetzungen in einem System, das zu jedem Zeitpunkt als eine einzige Phase angesehen werden darf.

Setzen wir zunächst das System als „geschlossen“ voraus, so läßt sich für jede Reaktion eine „Reaktionslaufzahl“ $\xi_{r}$ folgendermaßen definieren:

$$
\mathrm{d}_{r} m_{i}=M_{i} \mathrm{~d}_{r} n_{i}=M_{i} v_{i r} \mathrm{~d} \xi_{r},
$$

worin $v_{i r}$ der stöchiometrische Koeffizient des Stoffes $i$ in der Reaktion $r$ und $\mathrm{d}_{r} \boldsymbol{m}_{i}$ bzw. $\mathrm{d}_{r} n_{i}$ die infinitesimale Änderung der Masse bzw. Molzahl des Stoffes $i$ durch Ablauf der Reaktion $r$ ist.

Die „Affinität“ $A_{r}$ ist nach de Donder ${ }^{1}$ :

$$
A_{r} \equiv-\sum_{i} v_{i r} \bar{\mu}_{i}
$$

wobei die Summation so auszuführen ist, daß die stöchiometrischen Koeffizienten der entstehenden Stoffe positiv und die der reagierenden Stoffe negativ gezählt werden. Die „Reaktionsgeschwindigkeit“ $w_{r}$ ist definiert durch die Ableitung der Reaktionslaufzahl nach der Zeit:

$$
w_{r} \equiv \frac{\mathrm{d} \xi_{r}}{\mathrm{~d} t}
$$

17 R. D e f a y, Bull. Acad. roy. Belgique (Cl. Sc.) 15, 678 [1929]. 
Im wahren (ungehemmiten) thermodynamischen Gleichgewicht gilt: $A_{r}=0, w_{r}=0$.

Der weiteren Betrachtung legen wir sogleich den allgemeineren Fall zugrunde, bei dem $R$ Reaktionen gleichzeitig ablaufen und das System „offen“ ist, so $\mathrm{da}$ Stoffaustausch mit der Umgebung erfolgt. Ist $\mathrm{d}_{a} n_{i}$ die infinitesimale Änderung der Molzahl des Bestandteils $i$ durch Zufuhr von außen, so ergibt die Massenbilanz für jeden Stoff $(N=$ Zahl der Bestandteile):

$$
\mathrm{d} n_{i}=\sum_{r=1}^{R} v_{i r} \mathrm{~d} \xi_{r}+\mathrm{d}_{a} n_{i} \quad(i=1,2, \ldots N)
$$

Wir benötigen nun eine Formulierung des Energiesatzes für offene Systeme. Da in unserem Falle elektrische Vorgänge usw. nicht berücksichtigt werden sollen, besteht die äußere Arbeit nur in Volumenarbeit. Wäre das uniforme System geschlossen, so könnten wir schreiben, da mechanisches Gleichgewicht zu jedem Zeitpunkt vorausgesetzt ist:

$$
\mathrm{d} Q=\mathrm{d} U+p \mathrm{~d} V,
$$

worin $\mathrm{d} Q$ das Differential der dem System zugeführten Wärme ist. Bei offenen Systemen ist die Gesamtmasse $m$ variabel, und daher gilt Gl. (10) nicht mehr. Denken wir uns nun aus dem homogenen offenen System zu jedem Zeitpunkt einen Teil der Masse $m=1$ ausgeschnitten, so beträgt die diesem Teilsystem zugeführte Wärme den $m$-ten Teil der Gesamtwärme und kann nur zur Änderung der spezifischen inneren Energie $\bar{U}$ und des spezifischen Volumens $\bar{V}$ beitragen. Wir erhalten somit an Stelle von Gl. (10):

$$
\frac{1}{m} \mathrm{~d} Q=\mathrm{d} \bar{U}+p \mathrm{~d} \bar{V}
$$

Es gilt definitionsgemäß:

$$
U=m \bar{U}, \quad V=m \bar{V}, \quad \bar{H}=\bar{U}+p \bar{V},
$$

worin $\bar{H}$ die spezifische Enthalpie ist.

Aus (11) und (12) ergibt sich die Energiebilanzgleichung für eine offene Phase, die zuerst von Prigogin $\mathrm{e}^{5}$ angegeben wurde:

$$
\mathrm{d} Q=\mathrm{d} U+p \mathrm{~d} V-\bar{H} \mathrm{~d} m .
$$

Um die entsprechende Entropieänderung des Systems zu erhalten, gehen wir von der Gibbsschen Fundamentalgleichung (1) aus und benutzen die Massenbilanz (9) sowie die Energiebilanz (13). Wenn wir berücksichtigen, daß für eine infinitesimale Änderung, $\mathrm{d}_{a} m_{i}$, der Masse des Stoffes $i$ durch Zufuhr von außen gilt:

und

$$
M_{i} \mathrm{~d}_{\mathrm{a}} n_{i}=\mathrm{d}_{\mathrm{a}} m_{i}
$$

$$
\mathrm{d} m=\sum_{i=1}^{N} \mathrm{~d}_{\mathrm{a}} m_{i}
$$

so folgt aus (1), (2), (3), (7), (8), (9) und (13) die „Entropiebilanzgleichung“" $\left[\mathrm{Pr}\right.$ ig o g i n $\mathrm{e}^{5}$ ]:

$$
\frac{\mathrm{d} S}{\mathrm{~d} t}=\frac{\mathrm{d}_{\mathrm{a}} \mathrm{S}}{\mathrm{d} t}+\frac{\mathrm{d}_{\mathrm{i}} \mathrm{S}}{\mathrm{d} t},
$$

worin (Index a von „außen“, i von ,innen“) die „Entropieströmung“:

$$
\frac{\mathrm{d}_{\mathrm{a}} \mathrm{S}}{\mathrm{d} t}=\frac{1}{T} \frac{\mathrm{d} Q}{\mathrm{~d} t}+\frac{1}{T} \sum_{i=1}^{N}\left(\bar{H}-\mu_{j}\right) \frac{\mathrm{d}_{\mathrm{a}} m_{i}}{\mathrm{~d} t}
$$

und die „Entropieerzeugung“:

$$
\frac{\mathrm{d}_{\mathrm{i}} S}{\mathrm{~d} t}=\frac{1}{T} \sum_{r=1}^{R} A_{r} w_{r} \equiv \Theta .
$$

Setzen wir in obigen Gleichungen $\mathrm{d}_{\mathrm{a}} m_{i} / \mathrm{d} t=0$ $(i=1,2, \ldots N)$, so erhalten wir die entsprechenden Ausdrücke für ein geschlossenes System.

b) Wärmestrom und Entropiestrom

Für unsere späteren Betrachtungen sind folgende Umformungen von grundsätzlicher Bedeutung.

Es gilt $\left(H_{i}=\right.$ partielle Enthalpie des Stoffes $i$, $W_{p_{r}}=$ Reaktionswärme bei konstantem Druck für die Reaktion $r$ ):

$$
\begin{gathered}
\bar{H}=\sum_{i=1}^{N} x_{i} H_{j}, \\
\mathrm{~d} \bar{H}=\frac{\partial \bar{H}}{\partial p} \mathrm{~d} p+\frac{\partial \bar{H}}{\partial T} \mathrm{~d} T+\sum_{i=1}^{N} H_{i} \mathrm{~d} x_{i}, \\
W_{p_{r}}=-\sum_{i} M_{i} v_{i r} H_{i},
\end{gathered}
$$

wobei die letzte Summation über alle an der Reaktion beteiligten Stoffe im gleichen Sinne wie in Gl. (7) zu erstrecken ist.

Aus (4), (9) und (14) ergibt sich:

$\mathrm{d} x_{i}=\mathrm{d}\left(\frac{m_{i}}{m}\right)=\frac{1}{m} \sum_{r=1}^{R} M_{i} v_{i r} \mathrm{~d} \xi_{r}+\frac{1}{m}\left(\mathrm{~d}_{\mathrm{a}} m_{i}-x_{i} \mathrm{~d} m\right)$.

Aus (11), (12) und (19) bis (22) folgt schließlich:

$$
\mathrm{d} Q=\mathrm{d}_{\mathrm{m}} Q+\mathrm{d}_{\mathrm{z}} Q,
$$

worin das Differential der dem homogenen System bei konstantem $p, T, \xi_{r}$ durch Materieaustausch mit c.'c: Umgebung zugeführten Wärme:

$$
\mathrm{d}_{\mathrm{m}} Q=\sum_{i=1}^{N}\left(H_{i}-\bar{H}\right) \mathrm{d}_{\mathrm{a}} m_{i}
$$


und das Differential der dem System bei Verschwinden des Massenaustauschs zugeführten Wärme ${ }^{18}$ :

$\mathrm{d}_{\mathrm{z}} Q=\left(\frac{\partial H}{\partial p}-V\right) \mathrm{d} p+\frac{\partial H}{\partial T} \mathrm{~d} T-\sum_{r=1}^{R} W_{p_{\tau}} \mathrm{d} \xi_{r}$.

Aus (13), (15), (23) und (24) erhalten.wir:

$$
\mathrm{d}_{\mathrm{z}} Q+\sum_{i=1}^{N} H_{i} \mathrm{~d}_{\mathrm{a}} m_{i}=\mathrm{d} U+p \mathrm{~d} V .
$$

Die Gln. (17), (23) und (24) ergeben:

$$
\frac{\mathrm{d}_{\mathrm{a}} \mathrm{S}}{\mathrm{d} t}=\frac{1}{T} \frac{\mathrm{d}_{\mathrm{z}} Q}{\mathrm{~d} t}+\sum_{i=1}^{N} S_{i} \frac{\mathrm{d}_{\mathrm{a}} m_{j}}{\mathrm{~d} t},
$$

wobei die Beziehung

$$
\mu_{i}=H_{i}-T S_{i}
$$

benutzt wurde und $S_{i}$ die partielle Entropie des Stoffes $i$ bedeutet.

Die Gln. (23) bis (27) wurden zuerst von Prig og in $\mathrm{e}^{5}$ abgeleitet.

Wir nennen die Größe dQ/dt den „Wärmestrom“ (aus der Umgebung) und den Ausdruck $\mathrm{d}_{\mathrm{a}} m_{\mathrm{i}} / \mathrm{d} t$ den „Materiestrom der Komponente $i^{\text {" }}$ (aus der Umgebung). Dann liegt es nahe, die Größe [s. (23), (24), (26)]:

$$
\begin{aligned}
\frac{\mathrm{d}_{z} Q}{\mathrm{~d} t} & =\frac{\mathrm{d} U}{\mathrm{~d} t}+p \frac{\mathrm{d} V}{\mathrm{~d} t}-\sum_{i=1}^{N} H_{i} \frac{\mathrm{d}_{\mathrm{a}} m_{i}}{\mathrm{~d} t} \\
& =\frac{\mathrm{d} Q}{\mathrm{~d} t}-\sum_{i=1}^{N}\left(H_{j}-\bar{H}\right) \frac{\mathrm{d}_{\mathrm{a}} m_{j}}{\mathrm{~d} t}
\end{aligned}
$$

als „reduzierten Wärmestrom" zu bezeichnen; denn dieser Ausdruck stellt offensichtlich die Differenz zwischen dem gesamten Wärmestrom und demjenigen Teil desselben dar, der bei konstanten Werten des Druckes, der Temperatur und der Reaktionslaufzahlen durch Materieströme verursacht wird.

Gl. (27) zeigt, daß der „Entropiestrom“ $\mathrm{d}_{\mathrm{a}} \mathrm{S} / \mathrm{d} t$ (aus der Umgebung) sich besonders einfach mit Hilfe des „reduzierten Wärmestromes“ ausdrücken läßt. Bei der Besprechung der polythermen und kontinuierlichen Systeme werden wir auf die Gln. (27) und (29) zurückkommen.

c) Entropieerzeugung und phänomenologische Ansätze

Der Zweite Hauptsatz der Thermodynamik besagt in seiner erweiterten Formulierung, daß zu jedem Zeitpunkt für die Entropieerzeugung bei irreversiblen
Vorgängen gilt:

$$
\Theta>0 \text {. }
$$

Gemäß Gl. (18) erhalten wir für die „Dissipationsfunktion" ${ }^{19}$ :

$$
T \Theta=\sum_{r=1}^{R} A_{r} w_{r},
$$

d. h. der Ausdruck $T \Theta$ besteht aus einer Summe von Produkten aus Reaktionsgeschwindigkeiten und zugehörigen Affinitäten. Im thermodynamischen Gleichgewicht verschwinden alle Geschwindigkeiten und Affinitäten einzeln:

$$
w_{r}=0, \quad A_{r}=0, \quad(r=1,2 \ldots R) .
$$

Somit wird dann

$$
\Theta=0 \text {. }
$$

Beschränken wir uns auf Gleichgewichtsnähe, so lauten die allgemeinsten Ansätze für die Beziehung zwischen Geschwindigkeiten und Affinitäten [d e D o n de r ${ }^{20}$ ]:

$$
w_{r}=\sum_{s=1}^{R} a_{r s} A_{s} \quad(r=1,2 \ldots R) .
$$

Diese linearen „phänomenologischen Ansätze“ enthalten auch den Fall der Kopplung verschiedener chemischer Reaktionen (über die Koeffizienten $a_{r s}$ für $r \neq s$ ), und es sind gerade die Aussagen über die Kopplungskoeffizienten, die in der thermodynamischphänomenologischen Theorie zu fruchtbaren Anwendungen führen.

Für die phänomenologischen Koeffizienten $a_{r s}$ gelten:

1. die Onsagerschen Reziprozitätsbeziehungen:

$$
a_{r s}=a_{s r} \quad(r, s=1,2 \ldots R),
$$

2. der erweiterte Zweite Hauptsatz, Gl. (30), d.h. infolge den Gln. (31) und (34) die Bedingung $\left[\mathrm{d} \in \mathrm{D}\right.$ ond e r $\left.{ }^{20}\right]$ :

$$
\sum_{r=1}^{R} \sum_{s=1}^{R} a_{r s} A_{r} A_{s}>0 .
$$

Wegen Gl. (35) ist die linke Seite von (36) eine quadratische Form, die gemäß (36) positiv definit ist. Eine quadratische Form ist dann und nur dann positiv

18 Index $\mathrm{m}$ von „Materieaustausch“ und Index $\mathrm{z}$ von „Zustanclsänderung“".

19 De D o n d e r 1, 20 nennt $T \Theta$,puissance d'irréversibilité“.

20 Th. de Donder, Bull. Acad. roy. Belgique (Cl. Sc.) 24, 15 [1938]. 
definit, wenn die Determinante ihrer Koeffizienten, die wir hier mit $\operatorname{det}\left(a_{r s}\right)$ bezeichnen wollen, mit sämtlichen Hauptminoren positiv ist. Wir schreiben also an Stelle von (36):

$$
a_{r r}>0, \quad a_{r r} a_{s s}-a_{r s}^{2}>0, \ldots \operatorname{det}\left(a_{r s}\right)>0 .
$$

\section{Polytherme Systeme}

a) Bilanzgleichungen

Der Einfachheit halber beschränken wir unsere Diskussion auf geschlossene Systeme, die aus zwei „Phasen " (Indizes ' und ") bestehen, die jeweils auf konstanter Temperatur ( $T^{\prime}$ bzw. $\left.T^{\prime \prime}\right)$ gehalten werden. Die Drucke und Konzentrationen sind im allgemeinen sowohl in beiden Phasen verschieden als auch zeitlich veränderlich, jedoch innerhalb einer Phase örtlich konstant ${ }^{21}$. Diese Bedingungen sind realisierbar, wenn die beiden verschieden temperierten Teile des Systems durch eine (ganz oder teilweise) stoffdurchlässige Wand oder eine enge Verbindungsstelle (Kapillare) getrennt sind. Wir betrachten die Möglichkeit des Ablaufs folgender irreversibler Prozesse: je eine chemische Reaktion in jeder Phase, Stoffübergänge und Wärmetransport zwischen beiden Phasen. Damit erfassen wir alle bekannten ,thermomechanischen Phänomene“ (K n u d s e n - Effekt, Thermoosmose, „fountain effect“ beim flüssigen Helium II usw.)*.

Wir behalten unsere früheren Symbole bei, verabreden aber, daß sich ungestrichene Größen auf das Gesamtsystem, einfach gestrichene Symbole auf die eine Phase und doppelt gestrichene Größen auf die andere Phase beziehen sollen. Der Satz von der Erhaltung der Masse ergibt also ${ }^{22}$ :

$$
\begin{gathered}
\mathrm{d}_{\mathrm{t}} m_{i}^{\prime}=-\mathrm{d}_{\mathrm{t}} m_{i}^{\prime \prime} \quad(i=1,2 \ldots N), \\
\mathrm{d} m^{\prime}=-\mathrm{d} m^{\prime \prime}=\sum_{i=1}^{N} \mathrm{~d}_{\mathrm{t}} m_{i}^{\prime}, \\
\mathrm{d} m_{i}^{\prime}=M_{i} v_{i}^{\prime} \mathrm{d} \xi^{\prime}+\mathrm{d}_{\mathrm{t}} m_{i}^{\prime} \\
\mathrm{d} m_{i}^{\prime \prime}=M_{i} v_{i}^{\prime \prime} \mathrm{d} \xi^{\prime \prime}+\mathrm{d}_{\mathrm{t}} m_{i}^{\prime \prime} .
\end{gathered}
$$

Bei der Anwendung des Satzes von der Erhaltung der Energie bedenken wir, daß einerseits jede der

* Isotherme Membranprozesse sind als Sonderfall $\left(T^{\prime}=T^{\prime \prime}\right)$ in den folgenden Betrachtungen enthalten. Lassen wir auch eine elektrische Potentialdifferenz zwischen den beiden Phasen zu, so schließen wir Phänomene wie Elektroosmose, Membrandiffusionspotential usw. ein. Vgl. A. J. S t a verm a n, Chem. Weekbl. 47, 1 [19.51]. beiden Phasen mit der Umgebung Wärme austauscht (zur Aufrechterhaltung der Temperatur $T^{\prime}$ bzw. $T^{\prime \prime}$ ) und daß andererseits ein ständiger Wärmestrom von einer Phase zur anderen fließt. Nennen wir die von der Umgebung an die Phase' bzw. " abgegebene Wärme $\mathrm{d}_{\mathrm{a}}{ }^{\prime} Q$ bzw. $\mathrm{d}_{\mathrm{a}}{ }^{\prime \prime} Q$ (= Differential der ,,äußeren Wärme") und die jeweils von der anderen Phase an die Phase ' bzw. " abgegebene Wärme $\mathrm{d}_{\mathrm{i}}{ }^{\prime} Q$ bzw. $\mathrm{d}_{\mathrm{i}}{ }^{\prime} Q$ (=Differential der ,inneren Wärme“), so erhalten wir folgende Beziehungen ${ }^{23}$ :

$$
\begin{gathered}
\mathrm{d} Q=\mathrm{d}_{\mathrm{a}}{ }^{\prime} Q+\mathrm{d}_{\mathrm{a}}{ }^{\prime \prime} Q, \\
\mathrm{~d}^{\prime} Q=\mathrm{d}_{\mathrm{a}}{ }^{\prime} Q+\mathrm{d}_{\mathrm{i}}{ }^{\prime} Q, \quad \mathrm{~d}^{\prime \prime} Q=\mathrm{d}_{\mathrm{a}}{ }^{\prime \prime} Q+\mathrm{d}_{i}^{\prime \prime} Q, \\
\mathrm{~d} Q=\mathrm{d} U+P^{\prime} \mathrm{d} V^{\prime}+P^{\prime \prime} \mathrm{d} V^{\prime \prime}, \\
\mathrm{d} U=\mathrm{d} U^{\prime}+\mathrm{d} U^{\prime \prime}, \\
\mathrm{d}^{\prime} Q=\mathrm{d} U^{\prime}+P^{\prime} \mathrm{d} V^{\prime}-\bar{H}^{\prime} \mathrm{d} m^{\prime}, \\
\mathrm{d}^{\prime \prime} Q=\mathrm{d} U^{\prime \prime}+P^{\prime \prime} \mathrm{d} V^{\prime \prime}-\bar{H}^{\prime \prime} \mathrm{d} m^{\prime \prime} .
\end{gathered}
$$

Die Gln. (41) und (42) sind definitionsgemäß richtig, die Gln. (43) und (44) eine Folge der klassischen Form des Ersten Hauptsatzes, während die Gln. (45) die für jede der beiden Phasen angeschriebenen Energiebilanzgleichungen für offene homogene Systeme darstellen [s. Gl. (13)].

Aus den Gln. (41) bis (45) folgt das bereits von Prigogin $e^{5}$ gefundene Resultat:

$$
\mathrm{d}_{i}^{\prime} Q+\bar{H}^{\prime} \mathrm{d} m^{\prime}=-\left(\mathrm{d}_{\mathrm{i}}^{\prime \prime} Q+\bar{H}^{\prime \prime} \mathrm{d} m^{\prime \prime}\right) .
$$

Für den Wärmeaustausch zwischen beiden Phasen gilt hier also nicht die Beziehung:

$$
\mathrm{d}_{\mathrm{i}}{ }^{\prime} Q=-\mathrm{d}_{\mathrm{i}}{ }^{\prime \prime} Q
$$

sondern Gl. (46), die besagt, daß bei gleichzeitigem Stoffaustausch der Ausdruck $\mathrm{d}_{\mathrm{i}} Q+\bar{H} \mathrm{~d} m$ die Rolle der „Wärmemenge“ mit der Eigenschaft „von der

21 Genauer gesagt: Der Druck- und Konzentrationsausgleich innerhalb einer Phase erfolgt schnell im Vergleich zum Materietransport durch das „Ventil“, das die beiden Phasen trennt.

22 Es sei daran erinnert, daß wir je eine chemische Reaktion in jeder Phase voraussetzen, die charakterisiert ist durch $\xi^{\prime}$ und $A^{\prime}$ bzw. $\xi^{\prime \prime}$ und $A^{\prime \prime}$. Der Index $t$ an Stelle des Index a in den Symbolen $\mathrm{d}_{\mathrm{t}} m_{i}^{\prime}$ usw. deutet darauf hin, daß die Massenänderungen durch Materietransport von einer Phase zur anderen zustande kommen.

23 Die besondere Lage der Striche in den Symbolen $\mathrm{d}^{\prime} Q, \mathrm{~d}^{\prime \prime} Q$ usw. soll daran erinnern, da $\mathrm{d} Q$ kein vollständiges Differential, also $Q$ keine Zustandsfunktion ist. 
einen Phase aufgenommene Wärme $=$ von der anderen Phase abgegebene Wärme" übernimmt.

Aus Gln. (39), (41), (42) und (46) ergibt sich:

$$
\mathrm{d} Q=\mathrm{d}^{\prime} Q+\mathrm{d}^{\prime \prime} Q+\left(\bar{H}^{\prime}-\bar{H}^{\prime \prime}\right) \mathrm{d} m^{\prime} .
$$

Zerlegen wir nun jede der Größen $\mathrm{d}^{\prime} Q$ und $\mathrm{d}^{\prime \prime} Q$ im Sinne der Gln. (23) - (25), so können wir an Hand der obigen Beziehung zeigen, daß die Energiebilanzgleichungen (45) für offene Phasen zu keinerlei Widerspruch mit bekannten Tatsachen führen. Betrachten wir z. B. eine flüssige Mischung (Phase'), zu der kontinuierlich eine Flüssigkeit aus denselben Komponenten, aber von anderer Zusammensetzung (Phase") bei konstantem Druck und konstanter Temperatur und unter Ausschluß chemischer Reaktionen hinzugegeben wird. Dann folgt aus (23), (24), (38) und (39), da $\mathrm{d} T=\mathrm{d} p=\mathrm{d} \xi_{r}=0$ :

$\mathrm{d}^{\prime} Q+\mathrm{d}^{\prime \prime} Q=\left(\overline{H^{\prime \prime}}-\overline{H^{\prime}}\right) \mathrm{d} m^{\prime}+\sum_{i=1}^{N}\left(H_{j}^{\prime}-H_{j}^{\prime \prime}\right) \mathrm{d}_{\mathrm{t}} m_{j}^{\prime}$.

Einsetzen dieser Beziehung in Gl. (46a) ergibt für das Differential der mit der Umgebung während des Mischungsvorgangs insgesamt ausgetauschten Wärme, d. h. der meßbaren Mischungswärme:

$$
\mathrm{d} Q=\sum_{i=1}^{N}\left(H_{i}^{\prime}-H_{i}^{\prime \prime}\right) \mathrm{d}_{\mathrm{t}} m_{i}^{\prime} .
$$

Bei idealen und athermischen Gemischen gilt: $H_{i}{ }^{\prime}=H_{i}{ }^{\prime \prime}$ $(i=1,2 \ldots N)$; hier folgt also, wie es sein $\mathrm{mu} ß \mathrm{~d} Q=0$.

Zur Aufstellung der Entropiebilanz schreiben wir die Gibbssche Fundamentalgleichung (1) bei Berücksichtigung von (2), (7) und (40) für jede Phase an:

$$
T^{\prime} \mathrm{d} S^{\prime}=\mathrm{d} U^{\prime}+P^{\prime} \mathrm{d} V^{\prime}-\sum_{i=1}^{N} \mu_{j}^{\prime} \mathrm{d}_{\mathrm{t}} m_{j}^{\prime}+A^{\prime} \mathrm{d} \xi^{\prime},
$$

$T^{\prime \prime} \mathrm{d} S^{\prime \prime}=\mathrm{d} U^{\prime \prime}+P^{\prime \prime} \mathrm{d} V^{\prime \prime}-\sum_{i=1}^{N} \mu_{i}^{\prime \prime} \mathrm{d}_{\mathrm{t}} m_{i}^{\prime \prime}+A^{\prime \prime} \mathrm{d} \xi^{\prime \prime}$

Es gilt ferner:

$$
\mathrm{d} S=\mathrm{d} S^{\prime}+\mathrm{d} S^{\prime \prime}
$$

Somit erhalten wir aus (38), (39), (42), (45), (46) und (47) die „Entropiebilanzgleichung“,, d. h. die Gleichung für die Geschwindigkeit der Entropieänderung des gesamten (geschlossenen), aus den Phasen ' und " bestehenden Systems [Prigog in $\mathrm{e}^{5}$ ]:

$$
\frac{\mathrm{d} S}{\mathrm{~d} t}=\frac{\mathrm{d}_{\mathrm{a}} \mathrm{S}}{\mathrm{d} t}+\frac{\mathrm{d}_{\mathrm{i}} \mathrm{S}}{\mathrm{d} t},
$$

worin die „Entropieströmung“:

$$
\frac{\mathrm{d}_{\mathrm{a}} \mathrm{S}}{\mathrm{d} t}=\frac{1}{T^{\prime}} \frac{\mathrm{d}_{\mathrm{a}}^{\prime} Q}{\mathrm{~d} t}+\frac{1}{T^{\prime \prime}} \frac{\mathrm{d}_{\mathrm{a}}^{\prime \prime} Q}{\mathrm{~d} t}
$$

und die „Entropieerzeugung“:

$$
\begin{aligned}
& \frac{\mathrm{d}_{\mathrm{i}} S}{\mathrm{~d} t}=X_{Q}^{* *} K_{Q}-\sum_{i=1}^{N} X_{i} K_{i}^{* *}+\frac{A^{\prime}}{T^{\prime}} w^{\prime}+\frac{A^{\prime \prime}}{T^{\prime \prime}} w^{\prime \prime} \\
& =X_{Q}{ }^{*} K_{Q}-\sum_{i=1}^{N} X_{j} K_{i}^{*}+\frac{A^{\prime}}{T^{\prime}} w^{\prime}+\frac{A^{\prime \prime}}{T^{\prime \prime}} w^{\prime \prime} \equiv \Theta .
\end{aligned}
$$

In Gl. (51) wurde zur Abkürzung gesetzt:

$$
\begin{aligned}
& K_{Q}=\frac{1}{T^{\prime}}-\frac{1}{T^{\prime \prime}}, \\
& X_{Q}^{* *}=\frac{\mathrm{d}_{i}^{\prime} Q}{\mathrm{~d} t}+\bar{H}^{\prime} \frac{\mathrm{d} m^{\prime}}{\mathrm{d} t}=-\left(\begin{array}{c}
\mathrm{d}_{\mathrm{i}}{ }^{\prime \prime} Q \\
\mathrm{~d} t
\end{array}+\bar{H}^{\prime \prime} \frac{\mathrm{d} m^{\prime \prime}}{\mathrm{d} t}\right), \\
& X_{Q}^{*}=\frac{\mathrm{d}_{\mathrm{i}}^{\prime} Q}{\mathrm{~d} t}, \\
& K_{i}^{* *}=\frac{\mu_{i}^{\prime}}{T^{\prime}}-\frac{\mu_{i}^{\prime \prime}}{T^{\prime \prime}}, \\
& K_{i}^{*}=K_{i}^{* *}-\overline{H^{\prime}} . K_{Q}, \quad \quad(i=1,2 \ldots N) \\
& X_{i}=\frac{\mathrm{d}_{\mathrm{t}} m_{i}^{\prime}}{\mathrm{d} t}=-\frac{\mathrm{d}_{\mathrm{t}} m_{i}^{\prime \prime}}{\mathrm{d} t} \\
& w^{\prime}=\frac{\mathrm{d} \xi^{\prime}}{\mathrm{d} t}, \quad w^{\prime \prime}=\frac{\mathrm{d} \xi^{\prime \prime}}{\mathrm{d} t} .
\end{aligned}
$$

b) Wärmestrom

Da unser polythermes System voraussetzungsgemäß geschlossen ist, enthält die Entropieströmung gemäß Gl. (50) nur die mit der Umgebung (also mit zwei Wärmebehältern der Temperaturen $T^{\prime}$ und $T^{\prime \prime}$ ) ausgetauschte Wärme. Wir werden daher die Bezeichnungen „Wärmestrom“ und „Materiestrom“ auf die Größen $X_{Q}^{*}$ bzw. $X_{Q}^{* *}$ und $X_{i}$ anwenden, die sich auf den Wärme- und Materietransport zwischen den beiden Phasen des Systems beziehen und somit naturgemäß nicht mehr - wie bei den offenen homogenen Systemen - in der Entropieströmung, sondern in der Entropieerzeugung auftreten. Im übrigen gilt aber für die jeder Phase zugeführte Wärme dieselbe Zerlegung, wie sie in den Gln. (23) bis (25) durchgeführt wurde. Wir können also insbesondere aus dem „reinen Wärmestrom“

$$
X_{Q}^{*}=\frac{\mathrm{d}_{\mathrm{i}}{ }^{\prime} Q}{\mathrm{~d} t}
$$

nach dem Vorbilde von Gl. (29) den mit den „Materieströmen“"

$$
X_{i}=\frac{\mathrm{d}_{\mathrm{t}} m_{i}{ }^{\prime}}{\mathrm{d} t}=-\frac{\mathrm{d}_{\mathrm{t}} m_{i}{ }^{\prime \prime}}{\mathrm{d} t}
$$

gekoppelten Anteil abspalten und gelangen so $\mathrm{zu}$ 
einem „reduzierten Wärmestrom“

$X_{Q}=X_{Q}^{*}+\sum_{j=1}^{N}\left(\bar{H}^{\prime}-H_{i}^{\prime}\right) X_{i}=X_{Q}^{* *}-\sum_{i=1}^{N} H_{i}^{\prime} X_{j}$.

Auf Grund der Eigenschaft, die durch Gl. (46) beschrieben wird, können wir $X_{Q}^{* *}$ als „kommutativen Wärmestrom" bezeichnen ${ }^{24}$.

Für die weitere Durchführung der Theorie ist es prinzipiell gleichgültig, welchen der drei „Wärmeströme" man benutzt. P r i g o g i n e ${ }^{5}$ wählt die Größe $X_{Q}^{*}$, de Groot ${ }^{25}$ die Größe $X_{Q}^{* *}$. Wir werden jedoch zeigen, daß es am vorteilhaftesten ist, den „reduzierten Wärmestrom“ $X_{Q}$ zugrunde zu legen.

c) Entropieerzeugung und phänomenologische Ansätze

Zunächst gilt - wie früher - die Vorzeichenaussage für irreversible Prozesse:

$$
\Theta>0 \text {, }
$$

die in diesem Falle sogar aus der klassischen Form des Zweiten Hauptsatzes ableitbar ist ${ }^{26}$.

Für die Gültigkeit der linearen phänomenologischen Ansätze müssen wir wieder Gleichgewichtsnähe fordern. Es ist also z. B.

worin

$$
|\Delta T| \ll T,
$$

Dann gilt:

$$
T \equiv T^{\prime}, \quad \Delta T \equiv T^{\prime \prime}-T^{\prime} .
$$

$$
\frac{1}{T^{\prime}}-\frac{1}{T^{\prime \prime}}=\frac{\Delta T}{T^{2}} \text {. }
$$

Wir setzen:

$$
\frac{\mu_{i}^{\prime \prime}}{T^{\prime \prime}}-\frac{\mu_{i}^{\prime}}{T^{\prime}} \equiv \Delta\left(\frac{\mu_{i}}{T}\right)
$$

und schreiben zur Abkürzung:

$$
T K_{Q} \equiv L_{Q}, \quad-T K_{i}^{*} \equiv L_{i}^{*}, \quad-T K_{i}^{* *} \equiv L_{i}^{* *} .
$$

Somit erhalten wir aus (51), (52), (55), (56) und (64), wenn wir der Einfachheit halber die chemischen Reaktionen nicht berücksichtigen:

24 Wie de Gro ot 25 zeigt, ist $X_{Q}^{* *}$ gleich der Änderungsgeschwindigkeit desjenigen Teils der inneren Energie, der zwischen den beiden Phasen ausgetauscht wird, also gleich dem „Energiestrom“.

25 S. R. de Groot, L. Jansen u. P. Mazur, Physica 16, 691 [1950].

26 Dies liegt daran, daß unser polythermes System geschlossen ist. Es gilt also die Ungleichung von Cla us ius:

$$
\mathrm{d} S>\frac{\mathrm{d}_{\mathrm{a}}{ }^{\prime} \mathrm{Q}}{T^{\prime}}+\frac{\mathrm{d}_{\mathrm{a}}{ }^{\prime \prime} Q}{T^{\prime \prime}},
$$

woraus mit Gl. (49) und (50) die Beziehung (60) folgt.

$$
T \Theta=X_{Q}^{* *} L_{Q}+\sum_{i=1}^{N} X_{i} L_{i}^{* *}=X_{Q}^{*} L_{Q}+\sum_{i=1}^{N} X_{i} L_{i}^{*},
$$

worin

$$
\begin{aligned}
L_{Q}= & \frac{\Delta T}{T}, \\
L_{i}^{* *}=T \Delta\left(\frac{\mu_{i}}{T}\right) & \quad(i=1,2 \ldots N), \\
L_{i}^{*}= & T \Delta\left(\frac{\mu_{i}}{T}\right)+\bar{H}^{\prime} \frac{\Delta T}{T}
\end{aligned}
$$

und die $X$ durch die Gln. (53), (54) und (57) gegeben sind.

Wir können die $X$ als generalisierte „Ströme“ und die $L$ als generalisierte „Kräfte“, die diese Ströme treiben, bezeichnen. Gl. (66) ist also völlig dem Ausdruck (31) für homogene Systeme mit chemischen Reaktionen analog, wobei die "Ströme“ den Reaktionsgeschwindigkeiten und die „Kräfte“ den Affinitäten entsprechen. Im thermodynamischen Gleichgewicht verschwinden gemäß (53), (54), (57), (67), (68) und (69) sämtliche „Ströme“ und „Kräfte“ einzeln, so daß auch hier gilt: $\Theta=0$. Den Ausdruck $T \Theta$ bezeichnen wir - wie früher - als ,Dissipationsfunktion“.

Die allgemeinsten Ansätze für Gleichgewichtsnähe bestehen wiederum in homogenen linearen Beziehungen zwischen den Strömen und Kräften.

Wir erkennen nun bei Betrachtung der Gln. (66) bis (69) folgenden Umstand. Wenn als „Wärmestrom“ die Größe $X_{Q}^{*}$ oder $X_{Q}^{* *}$ benutzt wird, enthalten die „Kräfte“ für die Materieströme $\left(L_{i}^{*}\right.$ oder $\left.L_{i}^{* *}\right)$ noch den Ausdruck $\Delta T / T$, also die den „Wärmestrom“

${ }^{27}$ Es ist

$$
\left(\mu_{i}^{\prime \prime}-\mu_{i}^{\prime}\right)_{T} \equiv\left(\Delta \mu_{i}\right)_{T}=V_{i}^{\prime} \Delta p+\sum_{k=1}^{N-1}\left(\frac{\partial \mu_{i}}{\partial x_{k}}\right)_{T, p}^{\prime} \Delta x_{k},
$$

worin $\Delta p \equiv p^{\prime \prime}-p^{\prime}, \quad \Delta x_{i} \equiv x_{i}^{\prime \prime}-x_{i}^{\prime}$ und $V_{i}^{\prime}=$ partielles Volumen.

Im allgemeinen fordert die Voraussetzung der Gleichgewichtsnähe, daß neben $\Delta T$ auch $\Delta p$ und die $\Delta x_{k}$ klein sein müssen. Sind aber semipermeable Wände vorhanden, so können $\Delta p$ und die $\Delta x_{k}$ auch große Werte haben, wenn diese nur in der Nähe der für das osmotische Gleichgewicht gültigen Werte liegen. Sind die beiden Teilsysteme verschiedene "Phasen“ im Sinne der gewöhnlichen Thermodynamik (die dann auch frei aneinander grenzen können), so verliert die obige Beziehung ihre Gültigkeit, da hier im allgemeinen für das (nicht-osmotische) Gleichgewicht gilt:

$\Delta T=0, \quad \Delta p=0, \quad \Delta u_{i}=0, \quad \Delta x_{i} \neq 0 \quad(i=1,2, \ldots N)$. Alle folgenden Ausführungen sind nur sinnvoll, wenn die beiden homogenen Teile des Systems bei Gleichgewicht (nach Entfernung einer eventuellen Membran) zu einer einzigen Phase verschmelzen. 
treibende „Kraft“ $\left(L_{Q}\right)$. Dadurch treten zweierlei Komplikationen ein: 1. Die phänomenologischen Ansätze führen bei Zwei- und Mehrstoffgemischen zu sehr unhandlichen Ausdrücken. 2. Die „Wärmeleitfähigkeit", definiert als Koeffizient von $\Delta T$ in der expliziten Gleichung für den Wärmestrom, ist nicht in jedem Falle positiv.

Diese Schwierigkeiten werden behoben, wenn wir den ,reduzierten Wärmestrom “ $X_{Q}$ einführen. Mit der thermodynamischen Beziehung für Gleichgewichtsnähe ${ }^{27}$ [vgl. (61)—(64)]

$$
T \Delta\left(\frac{\mu_{i}}{T}\right)=\left(\Delta \mu_{i}\right)_{T}-\frac{H_{i}^{\prime}}{T} \Delta T
$$

folgt aus (59) und (66) bis (69) für die Dissipationsfunktion:

worin

$$
T \Theta=X_{Q} L_{Q}+\sum_{i=1}^{N} X_{j} L_{i},
$$

$$
L_{i}=\left(\Delta \mu_{i}\right)_{T} \quad(i=1,2 \ldots N)
$$

Die „Kräfte“ für die Materieströme $\left(L_{i}\right)$ sind jetzt nur noch Funktionen der Druck- und Konzentrationsdifferenzen. Über die mit dem „reduzierten Wärmestrom " definierten Wärmeleitfähigkeiten werden wir im nächsten Abschnitt berichten.

Ersetzen wir in den Gleichungen von Prigog in $\mathrm{e}^{5}$ bzw. de Groot ${ }^{25}$ den Wärmestrom $X_{Q}^{*}$ bzw. $X_{Q}^{* *}$ durch den reduzierten Wärmestrom $X_{Q}$, so haben wir an die Stelle von $L_{i}^{*}$ bzw. $L_{i}^{* *}$ die einfachere Größe $L_{i}$ zu setzen. Dies führt praktisch zu dem Ergebnis, daß in den Endformeln von Prigogine bzw. de Groot gewisse Terme, die neben Kombinationen von phänomenologischen Koeffizienten stehen und den Ausdruck $\overline{H^{\prime}}-H_{i}{ }^{\prime}$ bzw. $H_{i}{ }^{\prime}$ enthalten, zum Verschwinden gebracht werden. Dadurch wird die Theorie bedeutend übersichtlicher, ohne daß ihr Inhalt geändert wird 28 .

Der Übergang von einem System von Strömen und Kräften zu einem zweiten ergibt keine Änderungen in den Aussagen unserer Theorie, wenn die Ströme und Kräfte stets so bestimmt werden, daß die Summe aus allen Produkten der Form „Strom mal zugehörige Kraft“ und damit die Entropieerzeugung invariant gegenüber dieser Transformation ist. Die Wahl eines neuen Stromes z. B. hat immer so zu erfolgen, daß dieser eine homogene lineare Funktion der alten Ströme ist [s. Gl. (59)]. Dann transformieren sich auch die Kräfte [s. Gln. (68), (69) und (71)] und die phänomenologischen Koeffizienten homogen und linear [vgl. hierzu M e ix n e r ${ }^{4}$ ].

Die phänomenologischen Ansätze gemäß Gl. (70) lauten:

28 Die neuen phänomenologischen Koeffizienten sind nämlich von den ursprünglichen verschieden.

$$
\begin{gathered}
X_{i}=\sum_{k=1}^{N} a_{i k} L_{k}+b_{i} L_{Q}, \quad(i=1,2 \ldots N), \\
X_{Q}=\sum_{i=1}^{N} b_{i}^{\prime} L_{i}+c L_{Q} .
\end{gathered}
$$

Die Onsagerschen Reziprozitätsbeziehungen besagen:

$$
\begin{aligned}
a_{i k} & =a_{k i}, \\
b_{i} & =b_{i}{ }^{\prime} .
\end{aligned}
$$

Die Gln. (73a) beziehen sich auf die gegenseitige Kopplung der isothermen Materieströme, während die Gln. (73b) die typischen Aussagen für die Verknüpfung der „thermomechanischen Phänomene" mit den entsprechenden Umkehreffekten ergeben.

Der positiv definite Charakter der lokalen Entropieerzeugung [s. (60)] führt mit (70), (72) und (73) zu folgenden Vorzeichenaussagen [vgl. (37)]:

$$
\begin{gathered}
a_{i i}>0, \quad c>0, \quad a_{i i} a_{k k}-a^{2}{ }_{i k}>0, \\
a_{i i} c-b_{i}{ }^{2}>0, \ldots \operatorname{det}\left(a_{i k}\right)>0, \Delta>0 .
\end{gathered}
$$

Hierin ist $\Delta$ die Determinante der Koeffizienten der quadratischen Form $T \Theta$ :

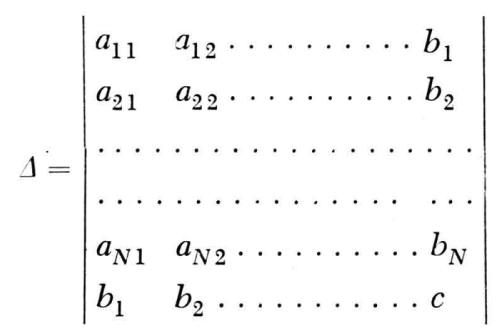

\section{d) Wärmele itfähigke it}

Wir unterscheiden die „Wärmeleitfähigkeit für homogene Durchmischung“ $\lambda$ und die „Wärmeleitfähigkeit für den stationären Zustand“ $\varkappa$. Bereits Meixner ${ }^{4}$ hat auf die Wichtigkeit dieser Unterscheidung bei Überlagerungsprozessen aufmerksam gemacht. Wir wollen zeigen, daß 1. nur bei Zugrunde. legung des „reduzierten Wärmestromes“ physikalisch sinnvolle „Wärmeleitfähigkeiten“ resultieren, 2. diə Differenz $\lambda-\varkappa$ dann stets positiv und durch die übrigen phänomenologischen Koeffizienten in einfacher Weise ausdrückbar ist.

Infolge unserer Definition von $X_{Q}$ ist in Gl. (72 b) die Temperaturdifferenz $\Delta T$ nur in $L_{Q}$ enthalten; denn es ist gemäß Gl. (67) und (71):

$$
L_{Q}=\frac{\Delta T}{T}, \quad L_{i}=\left(\Delta \mu_{i}\right)_{T}, \quad(i=1,2 \ldots N) .
$$


Setzen wir $L_{i}=0(i=1,2 \ldots N)$, so verschwinden der Druckunterschied und alle Konzentrationsdifferenzen zwischen den beiden Phasen (,homogene Durchmischung"). Wir haben den Zustand „reiner Wärmeleitung“, und es gilt gemäß Gl. (72b) für den Wärmestrom:

$$
\left.X_{Q}=c L_{Q}=\frac{c}{T}\right\lrcorner T \text {. }
$$

Wir definieren daher die Größe

$$
\frac{c}{T} \equiv \lambda
$$

als „Wärmeleitfähigkeit für homogene Durchmischung“. Aus (74) folgt ${ }^{29}$ :

$$
\lambda>0 \text {. }
$$

Man überzeugt sich leicht davon, daß bei Wahl eines anderen Wärmestromes (z. B. $X_{Q}^{*}$ oder $X_{Q}^{* *}$ ) die

29 Die Aussage $\lambda>0$ bedeutet, daß der „Wärmestrom“ von der Phase höherer Temperatur zu derjenigen tieferer Temperatur fließt.

30 Wählt man z. B. $X_{Q}^{* *}$ als Wärmestrom, so lauten die phänomenologischen Ansätze gemäß Gl. (66):

$$
\begin{gathered}
X_{i}=\sum_{k=1}^{N} a_{i k} L_{k}^{* *}+\beta_{i} L_{Q} \quad(i=1,2 \ldots N), \\
X_{Q}^{* *}=\sum_{i=1}^{N} \beta_{i} L_{i}^{* *}+\gamma L_{Q},
\end{gathered}
$$

worin die $\alpha_{i k}, \beta_{i}$ und $\gamma$ neue phänomenologische Koeffizienten sind.

Es gilt nun infolge (59), (67), (68) und (71):

$$
L_{i}^{* *}=L_{i}-\frac{H_{i}^{\prime}}{T} \Delta T, \quad L_{i}=\left(\Delta u_{i}\right)_{T}, L_{Q}=\frac{\Delta T}{T},
$$

mithin:

$$
X_{Q}^{* *}=\sum_{i=1}^{N} \beta_{i}\left(\Delta u_{i}\right)_{T}+\frac{1}{T}\left(\gamma-\sum_{i=1}^{N} \beta_{i} H_{i}{ }^{\prime}\right) \Delta T .
$$

Die „Wärmeleitfähigkeit für homogene Durchmischung“ $\lambda^{\prime}$ beträgt also [vgl. de Groot ${ }^{6}$ ]:

$$
i^{\prime}=\frac{1}{T}\left(\gamma-\sum_{i=1}^{N} \beta_{i} H_{i}^{\prime}\right) \text {. }
$$

Die $\beta_{i}$ können nun sowohl positiv als auch negativ sein, so daß das Vorzeichen von $\lambda^{\prime}$ unbestimmt ist. - Bei $\mathrm{M}$ e ix n e ${ }^{4}$ tritt eine zusätzliche Komplikation dadurch auf, daß seine "chemischen Potentiale“, die wir $\mu_{i}^{*}$ nennen wcllen, nicht mit unseren $\mu_{i}$ identisch sind. Es gilt der Zusammenhang 11:

$$
\mu_{i}^{*}=!_{i}-\bar{F},
$$

worin $\bar{F}=\bar{U}-T \bar{S}$ die spezifische (Helmholtzsche) Freie Energie ist. Das thermodynamische Gleichgewicht zwischen zwei Phasen fordert bei uns: $\mu_{i}^{\prime}=\mu_{i}^{\prime \prime}$, bei Meixner jedoch:

$$
u_{i}^{* \prime}+\bar{F}^{\prime}=u_{i}^{* \prime \prime}+\bar{F}^{\prime \prime} .
$$

den Gln. (72 b) und (76) entsprechenden Beziehungen zu einer „Wärmeleitfähigkeit“ führen, deren Vorzeichen unbestimmt ist ${ }^{30}$.

$\mathrm{Da}$ aus physikalischen Gründen gefordert werden muß, daß eine Größe, die den Namen „Wärmeleitfähigkeit" trägt, stets positiv sein soll, erkennen wir, daß bei Kopplung von Wärme- und Stoffübergang nicht irgendein „Wärmestrom“, sondern gerade der "reduzierte Wärmestrom “ $X_{Q}$ diejenige Funktion ist, die zur Beschreibung des Wärmeübergangs zwischen verschieden temperierten Teilen des Systems am besten geeignet ist. Es ist sicher kein Zufall, daß auch in anderer Hinsicht die Benutzung des reduzierten Wärmestromes zu besonders einfachen Ergebnissen führt; dies wurde oben schon angedeutet und wird bei der Besprechung der kontinuierlichen Systeme durch weitere Beispiele belegt werden.

Der Zustand homogener Durchmischung ist indessen kein stationärer Zustand; denn gemäß Gl. (72a) erzeugt die vorgegebene Temperaturdifferenz $\Delta T$ sofort Materieströme („Thermoosmose“ und analoge „thermomechanische Phänomene"), die ihrerseits wieder Druck- und Konzentrationsdifferenzen aufbauen. Schließlich wird der Vorgang dadurch stationär, daß die Mengen der einzelnen Stoffe in den beiden Phasen und der Wärmestrom zeitlich konstant werden ${ }^{31}$. Da wir in den Gln. (72) chemische Reaktionen nicht berücksichtigt haben, wird der stationäre Zustand beschrieben durch

$$
X_{i}=0 \quad(i=1,2 \ldots N), \quad X_{Q}=\text { const } .
$$

Wir eliminieren nun aus den Gln. (72) die $L_{i}$.

Dazu setzen wir

$$
b_{i} \equiv \sum_{k=1}^{N} a_{i k} Q_{k} \quad(i=1,2 \ldots N) .
$$

Hieraus folgt durch Auflösen der Gl. (80) nach den $Q_{k}$ und nachfolgende Addition bei Berücksichtigung von (75):

$c-\sum_{i=1}^{N} b_{i} Q_{i}=c-\sum_{i=1}^{N} \sum_{k=1}^{N} a_{i k} Q_{i} Q_{k}=\frac{\lrcorner}{\operatorname{det}\left(a_{i k}\right)}$,

worin $\Delta$ die Determinante in Gl. (75) ist.

Aus den Gln. (72), (73) und (80) erhalten wir:

$$
X_{Q}=\sum_{i=1}^{N} Q_{i} X_{i}+\left(c-\sum_{i=1}^{N} b_{i} Q_{i}\right) L_{Q} .
$$

31 Die Stationaritätsbedingungen sind komplizierter, wenn außer $\Delta T$ auch $\Delta p$ konstant gehalten wird (vgl. hierzu die fclgende Mitteilung Z. Naturforschg. 6a, Heft 9 [1951]. 
Für den stationären Zustand folgt aus (79) und (82) mit $L_{Q}=\Delta T / T$ :

$$
X_{i}=0 \quad(i=1,2 \ldots N), \quad X_{Q}=\varkappa \Delta T,
$$

worin

$$
\varkappa \equiv \frac{1}{T}\left(c-\sum_{i=1}^{N} b_{i} Q_{i}\right)
$$

die „Wärmeleitfähigkeit für den stationären Zustand“ ist. Mit Gl. (81) und den Ungleichungen (74) ergibt sich $^{32}$ :

$$
x>0 \text {. }
$$

Aus (77) und (84) erhalten wir mit (74) und (81) die wichtige Beziehung:

$\lambda-\varkappa=\frac{1}{T} \sum_{i=1}^{N} b_{i} Q_{i}=\frac{1}{T} \sum_{i=1}^{N} \sum_{k=1}^{N} a_{i k} Q_{i} Q_{k}>0$.

Die $Q_{i}$ sind gemäß Gl. (80) auf die Minoren der Determinante in Gl. (75) und somit auf die phänomenologischen Koeffizienten $a_{i k}, b_{i}$ zurückführbar.

Es sei bemerkt, daß die $N$ Größen $Q_{i}$ gemäß Gl. (82) mit den „Überführungswärmen“ von Eastman ${ }^{33}$ und $\mathrm{W}$ a g $\mathrm{n}$ e ${ }^{34}$ identisch sind [vgl. hierzu M e ix $n$ er ${ }^{4}$ und de Groot ${ }^{6}$.

\section{Kontinuierliche Systeme}

a) Bilanzgleichungen

Bei kontinuierlichen Systemen müssen die Erhallungssätze der Physik (Erhaltung der Masse, Impulssatz, Energiesatz) und der Zweite Hauptsatz der Thermodynamik in Form lokaler Bilanzgleichungen formuliert werden, die für jedes Volumenelement gelten. Wir betrachten folgende irreversible Phänomene: Substanztransport mit innerer Reibung, Diffusion, Wärmeleitung, Elektrizitätsleitung, chemische Reaktionen und deren gegenseitige Überlagerungen. Dabei beschränken wir unsere Diskussion auf Prozesse in isotropen Medien bzw. solche Vorgänge in anisotropen Körpern, bei denen die Anisotropie nicht

32 Legt man einen anderen „Wärmestrom“ (z. B. $X_{Q}^{*}$ oder $\left.X_{Q}^{* *}\right)$, der eine homogene lineare Funktion der hier benutzten Ströme $\left(X_{Q}\right.$ und $\left.X_{i}\right)$ ist, zugrunde und definiert in analoger Weise wie oben eine „Wärmeleitfähigkeit für den stationären Zustanc “ $\varkappa^{\prime}$, so findet man wiederum: $\varkappa^{\prime}>0$. Daher geben auch M e ix ne ${ }^{4}$ und de Groot ${ }^{6}$, die nicht den "reduzierten Wärmestrom“ benutzen, die zu (84) und (85) analogen Beziehungen (bei kontinuierlichen Systemen) an.

(78) und (85) lassen sich auch ohne Benutzung der Reziprozitätsbeziehungen ableiten. in die Überlegungen eingeht (z. B. thermoelektrische Erscheinungen in Drähten).

Es sei $\varrho_{i}$ die Dichte des Stoffes $i, \varrho=\sum_{j=1}^{N} \varrho_{j}$ die Gesamtdichte, $\overrightarrow{u_{i}}$ die mittlere (makroskopische) Geschwindigkeit der Teilchen des Bestandteils $i$ und $\vec{v}$ die Schwerpunktsgeschwindigkeit des betrachteten Volumenelementes.

Dann gilt:

$$
\vec{v}=\sum_{i=1}^{N} x_{i} \vec{u}_{i}=\sum_{i=1}^{N} \frac{\varrho_{i}}{\varrho} \vec{u}_{i} .
$$

Den Vektor der Diffusionsstromdichte des Stoffes $i$, kurz „Diffusionsstrom“ genannt, bezeichnen wir mit $\vec{J}_{i}$. Er ist definiert durch:

$$
\overrightarrow{J_{i}}=\varrho_{i}\left(\overrightarrow{u_{i}}-\vec{v}\right)
$$

Es ergibt sich die Identität:

$$
\sum_{i=1}^{N} \vec{J}_{i}=0
$$

Es sei

$$
\frac{\mathrm{d}}{\mathrm{d} t}=\frac{\partial}{\partial t}=\vec{v} \text { grad }
$$

der Operator der „substantiellen Differentiation“, $w_{r}+$ die Reaktionsgeschwindigkeit (je Volumeneinheit) der chemischen Reaktion $r, \vec{K}_{j}$ die äußere Kraft je Masseneinheit, die auf die Moleküle des Stoffes $j$ wirkt, $\vec{W}^{*}$ der „Wärmestrom“ (= Vektor der Wärmestromdichte), $P$ der Drucktensor ${ }^{35}$ mit dem statischen Druck $p$ und den Reibungsdrucken $p_{i k}\left(=p_{k i}\right.$; $i, k=1,2,3), v_{1}, v_{2}, v_{3}$ die Komponenten von $\vec{v}$, $z_{1}, z_{2}, z_{3}$ die Raumkoordinaten.

Dann ergibt die Massenbilanz:

$$
\begin{aligned}
\varrho \frac{\mathrm{d} x_{i}}{\mathrm{~d} t} & =\frac{\partial \varrho_{i}}{\partial t}+\operatorname{div}\left(\varrho_{i} \vec{v}\right) \\
& =-\operatorname{div} \vec{J}_{i}+\sum_{r=1}^{R} v_{i r} M_{i} w_{r}^{+}(\mathrm{i}=1,2 \ldots N) .
\end{aligned}
$$

23 E. D. E a s tm a n, J. Amer. chem. Soc. 48, 1482 [1926]; 50, 283, 293 [1928].

34 C. W a g n e r, Ann. Physik (5) 3, 629 [1929]; 6, 370 [1930].

35 Wir schreiben den Drucktensor $P$ in folgender Form.

$$
P=\left(\begin{array}{lll}
p+p_{11} & p_{12} & p_{13} \\
p_{21} & p+p_{22} & p_{23} \\
p_{31} & p_{32} & p+p_{33}
\end{array}\right)
$$


Der Impulssatz fordert:

$$
\varrho \frac{\mathrm{d} \vec{v}}{\mathrm{~d} t}=\sum_{i=1}^{N} \varrho_{i} \vec{K}_{i}-\operatorname{Div} P,
$$

worin der Vektor Div $P$ die „Tensordivergenz" von $P$ ist ${ }^{36}$.

Der Energiesatz verlangt, wenn wir die vorige Gleichung berücksichtigen:

$$
\varrho \frac{\mathrm{d} \bar{U}}{\mathrm{~d} t}=\frac{\partial U^{+}}{\partial t}+\operatorname{div}\left(U^{+} \vec{v}\right)
$$

$=-\sum_{i=1}^{3} \sum_{k=1}^{3} p_{i k} \frac{\partial v_{i}}{\partial z_{k}}-p \operatorname{div} \vec{v}+\sum_{i=1}^{N} \vec{K}_{i} \vec{J}_{i}-\operatorname{div} \vec{W}^{*}$,

worin $U^{+}=\varrho \bar{U}$ die Dichte der inneren Energie ist.

Einsetzen dieser lokalen Bilanzgleichungen in die lokale Form der Gibbsschen Fundamentalgleichung, Gl. (5), liefert nach einigen Umrechnungen die „Kontinuitätsgleichung für die Entropie“ oder „Entropiebilanzgleichung“" $\left[\mathrm{E} \mathrm{ckart}^{3}, \mathrm{M}\right.$ e ix ner ${ }^{4}, \mathrm{Prigo-}$ gin $\mathrm{e}^{5}$ ]:

$$
\varrho \frac{\mathrm{d} \bar{S}}{\mathrm{~d} t}=\frac{\partial S^{+}}{\partial t}+\operatorname{div}\left(S^{+} \vec{v}\right)=-\operatorname{div} \vec{S}^{\prime}+\vartheta
$$

worin die „Entropiedichte“

$$
S^{+}=\varrho \bar{S},
$$

der (konvektionsfreie) „Entropiestrom“ (= Vektor der Entropiestromdichte)

$$
\vec{S}^{\prime}=\frac{1}{T}\left(\vec{W}^{*}-\sum_{i=1}^{N} \mu_{i} \vec{J}_{i}\right)
$$

und die „lokale Entropieerzeugung“

$$
\begin{array}{r}
\vartheta=-\frac{\vec{W}^{*}}{T^{2}} \operatorname{grad} T+\frac{1}{T} \sum_{i=1}^{N} \vec{J}_{i}\left(\vec{K}_{i}-T \operatorname{grad} \frac{\mu_{i}}{T}\right) \\
+\frac{1}{T} \sum_{r=1}^{R} A_{r} w_{r}^{+}-\frac{1}{T} \sum_{i=1}^{3} \sum_{k=1}^{3} p_{i k} \frac{\partial v_{i}}{\partial z_{k}} .
\end{array}
$$

Gl. (90) entspricht den Gln. (16) und (49). Der Vektor $S^{+} \vec{v}$ ist der konvektive Anteil und der Vektor $\vec{S}^{\prime}$ der nichtkonvektive Anteil des gesamten Entrop:estromes: $\vec{S}=S^{+} \vec{v}+\vec{S}^{\prime}$.

36 Die Komponenten dieses Vektors in Cartesischen Kocrdinaten sind:

$$
\frac{\partial p}{\partial z_{i}}+\sum_{k=1}^{3} \frac{\partial p_{i k}}{\partial z_{k}} \quad(i=1,2,3) .
$$

Der Term - div $\overrightarrow{S^{\prime}}$ entspricht der Größe $\mathrm{d}_{\mathrm{a}} S / \mathrm{d} t$ bei diskontinuierlichen Systemen, und $\vartheta$ ist dem Ausdruck $\Theta=\mathrm{d}_{\mathrm{i}} \mathrm{S} / \mathrm{d} t$ analog.

Die Gln. (90)-(93) gelten für beliebige (isotrope) fluide Medien ${ }^{37}$.

b) Reduzierter Wärmestrom

Den „Materieströmen“ $X_{j}$ bei polythermen Systemen entsprechen die Größen $\varrho_{j} \overrightarrow{u_{j}}$ bei kontinuierlichen Systemen, da deren Summe nicht verschwindet [s. Gl. (87) und (89)]. Nach dem Vorbild von Gl. (59) gelangen wir daher mit Gl. (87) zu der Größe

$$
\vec{W}^{* *}=\vec{W}^{*}+\varrho \vec{H} \vec{v},
$$

die dem „kommutativen Wärmestrom“ entspricht, und zu der Größe

$$
\vec{W}=\vec{W}^{*}-\sum_{j=1}^{N} \varrho_{j} H_{i} \overrightarrow{u_{i}},
$$

die dem ,reduzierten Wärmestrom“ entspricht.

Da nun $\varrho \bar{H}=\sum_{i=1}^{N} \varrho_{i} H_{j}$ ist, folgt aus den beiden letzten Gleichungen:

$$
\vec{W}=\vec{W}^{*}-\sum_{i=1}^{N} H_{i} \vec{J}_{i}
$$

In der Literatur werden noch weitere Größen als „Wärmestrom“ bzw. „Energiestrom“ benutzt. So verwendet $\mathrm{Eckart}^{3}$ den Ausdruck

$$
\vec{W}^{\prime}=\vec{W}^{*}-\sum_{i=1}^{N} u_{i} \vec{J}_{i}
$$

und $\mathrm{W}$ a ld $\mathrm{m}$ a n $\mathrm{n}^{38}$ die Größe

$$
\vec{W}^{\prime \prime}=\vec{W}^{*}+\varrho \bar{H} \quad(\vec{v}-\vec{u})=\vec{W}^{* *}-\varrho \vec{H} \vec{u},
$$

worin $\vec{u}$ die mittlere molare Geschwindigkeit des Volumenelementes ist ${ }^{39}$.

37 Die Beziehungen gelten sinngemäß auch für isotrope feste Körper, wenn man an Stelle der Reibungsclrucke und Geschwindigkeitskompcnenten die Spannungen und Dehnungen aus der Mechanik der deformierbaren festen Medien einführt.

s8 L. W a ld m a n n, Z. Naturforschg. 4 a, 105 [1949].

$$
{ }^{39} \text { Es gilt: } \vec{u}=\sum_{i=1}^{N} \vec{x}_{i} \overrightarrow{u_{i}}, \quad c(\vec{u}-\vec{v})=\sum_{i=1}^{N} \vec{J}_{j} / M_{i},
$$

worin $\overline{x_{i}}$ Molenbruch der Komponente $i$ und $c$ totale molare Volumenkonzentration. Der "Konvektionsstrom“ $\varrho \vec{v}$ läßt sich nicht durch die $\overrightarrow{\boldsymbol{J}}_{j}$ homogen und linear ausclrücken. 
Für die linearen phänomenologischen Ansätze können alle Ausdrücke als „Ströme“ benutzt werden, die homogene lineare Funktionen der in Gl. (93) auftretenden Größen $\vec{W}^{*}$ und $\vec{J}_{i}(i=1,2, \ldots N)$ sind [vgl. M e ix n e r $\left.{ }^{4}\right]$. Daher sind für die weitere Durchführung der Rechnung $\vec{W}^{*}, \vec{W}, \vec{W}^{\prime}$ und $\vec{W}^{\prime \prime}$, nicht aber $\vec{W}^{* *}$ verwendbar ${ }^{39}$.

Auf zwei Vorteile des „reduzierten Wärmestromes“ $\vec{W}$, die bereits Prig o g in $\mathrm{e}^{5,10}$ erkannt hat, sei schon an dieser Stelle hingewiesen:

1. Bei Zugrundelegung von $\vec{W}$ folgt aus Gln. (92) und (94):

$$
\overrightarrow{S^{\prime}}=\frac{\vec{W}}{T}+\sum_{i=1}^{N} S_{i} \vec{J}_{i}
$$

also ein besonders einfacher und plausibler Ausdruck für den Entropiestrom $\left(S_{i}=\right.$ partielle Entropie des Stoffes $i$ ). Gl. (95) ist der Gl. (27) vollkommen analog.

2. Bei Verwendung von $\vec{W}$ ergibt sich gemäß Gl. (94) für die Koeffizienten der Diffusionsströme $\overrightarrow{\boldsymbol{J}}_{i}$ in Gl. (93) der Ausdruck

$$
\vec{K}_{i}-\left(\operatorname{grad} \mu_{i}\right)_{T}
$$

in Analogie zu $\left(\Delta \mu_{i}\right)_{T}$ in Gln. (70) und (71). Diese Größe hat einen numerisch bestimmten Wert, da siẻ nur die Differentialquotienten $\partial \mu_{i} / \partial p=V_{\mathrm{i}}$ (partielles Volumen) und $\partial \mu_{i} / \partial x_{k}$ enthält. Dagegen ist der in Gl. (93) auftretende Ausdruck

$$
\vec{K}_{i}-T \operatorname{grad} \frac{\mu_{i}}{T}
$$

infolge der Beziehung

$$
\frac{\partial}{\partial T}\left(\frac{\mu_{i}}{T}\right)=-\frac{H_{i}}{T^{2}}
$$

nur bis auf eine additive Konstante bestimmt.

c) Lokale Entropieerzeugung und phänomenologische Ansätze

Die verallgemeinerte Formulierung des Zweiten Hauptsatzes verlangt, daß bei Ablauf von irreversiblen Prozessen für die lokale Entropieerzeugung $\vartheta$ gilt:

$$
\vartheta>0 \text {. }
$$

40 „Energiedissipation“ heißt nach M e ix ne r 4 die Dissipationsfunktion bei kontinuierlichen Systemen $(T \vartheta)$.

41 Ein solches ist nur bei Einwirkung äußerer Kräfte, z. B. der Schwerkraft, denkbar. Andernfalls wird das System im Gleichgewicht zu einem homogenen System.
Mit der thermodynamischen Beziehung

$$
T \operatorname{grad} \frac{\mu_{i}}{T}=\left(\operatorname{grad} \mu_{i}\right)_{T}-\frac{H_{i}}{T} \operatorname{grad} T
$$

folgt bei Benutzung des „reduzierten Wärmestromes“ $\vec{W}$ aus Gl. (93) und (94) für die „Energiedissipation" " 40 :

$$
\begin{array}{r}
T \vartheta=-\frac{\vec{W}}{T} \operatorname{grad} T+\sum_{i=1}^{N} \vec{J}_{j}\left[\vec{K}_{i}-\left(\operatorname{grad} \mu_{j}\right)_{T}\right] \\
+\sum_{r=1}^{R} A_{r} w_{r}^{+}-\sum_{i=1}^{3} \sum_{k=1}^{3} p_{i k} \frac{\partial v_{i}}{\partial z_{k}} .
\end{array}
$$

Bedenken wir, daß für ein kontinuierliches System im Gleichgewicht ${ }^{41}$ nach den Gesetzen der klassischen Thermodynamik gilt:

$$
\begin{gathered}
T=\text { const }, \\
\mu_{i}+\Phi_{i}=\text { const, }
\end{gathered}
$$

worin $\Phi_{i}$ das Potential des äußeren Kraftfeldes bezüglich der Komponente $i$ (bezogen auf die Masseneinheit) bedeutet, so folgt die Gleichgewichtsbedingung:

$$
\begin{aligned}
\vec{K}_{i}=-\operatorname{grad} \Phi_{i}=\operatorname{grad} \mu_{i} \quad(i=1,2 \ldots N), & \operatorname{grad} T=0 .
\end{aligned}
$$

Bei Beachtung dieser Beziehungen erkennt man, daß in der Energiedissipation, Gl. (98), alle "Ströme“" und „Kräfte“ einzeln verschwinden, wenn thermodynamisches Gleichgewicht herrscht; somit gilt dann auch: $\vartheta=0$. Hiermit sind wieder die Voraussetzungen für die Formulierung der phänomenologischen Ansätze und die Anwendbarkeit der Onsagerschen Reziprozitätsbeziehungen gegeben.

42. Der Umstand, daß bei Meixner ${ }^{4}$ im Gleichgewicht nicht alle $\vec{K}_{i}^{*}$ einzeln verschwinden, liegt wiederum an der Verwendung eines anderen "chemischen Potentials “. $\mathrm{Eckart}{ }^{3}$ und $\mathrm{Me}$ ixne ${ }^{4}$ benutzen außerdem einen anderen Wärmestrom $\left(\vec{W}^{\prime}\right.$ bzw. $\left.\vec{W}^{*}\right)$; dadurch werden die $\vec{K}_{i}^{*}$ von den unseren verschieden. Obige Ansätze entsprechen denen von Prig o g in e ${ }^{5}$. - Definiert man reversible Prozesse durch die Bedingung $\vartheta=0$, so sind außer dem gewöhnlichen thermodynamischen Gleichgewicht noch „reversible Bewegungen“ in Betracht zu ziehen. Verschwindet die Volumenviskosität, so gibt es reversible Bewegungen, die mit inneren Veränderungen des Systems verknüpft sind. In diesem Falle ist für $\vartheta=0$ gemäß (98), (100d) und (103) notwendig und hinreichend, daß $L_{11}=L_{22}=L_{33}$, $L_{12}=L_{23}=L_{31}=0$. Dies führt zu der Aussage, daß $\vartheta$ allgemein nicht positiv definit, sondern semidefinit ist. Vgl. hierzu M e ix ne r ${ }^{4}$. Wir sehen im folgenden von "reversiblen Bewegungen“ ab. 
Die „phänomenologischen Ansätze“, d. h. die homogenen linearen Beziehungen zwischen „Strömen“ und „Kräften“, lauten gemäß Gl. (98), wie zuerst $\mathrm{E} \mathrm{ckart^{3 }}$ und $\mathrm{Me}$ i x n e r ${ }^{4}$ gezeigt haben ${ }^{42}$ :

$$
\begin{gathered}
\vec{J}_{i}=\sum_{k=1}^{N} a_{i k} \vec{K}_{k}^{*}+\beta_{i} \vec{L}_{W} \quad(i=1,2, \ldots N), \\
\vec{W}=\sum_{i=1}^{N} \beta_{i}^{\prime} \vec{K}_{i}^{*}+\gamma \vec{L}_{W}, \\
w_{r}^{+}=\sum_{s=1}^{R} a_{r s} A_{s} \quad(r=1,2, \ldots R), \\
p_{i k}=2 \eta L_{i k}-\frac{2}{3} \eta \delta_{i k}\left(L_{11}+L_{22}+L_{33}\right), \\
\delta_{i k}=\left\{\begin{array}{l}
0 \text { für } i \neq k \\
1 \text { für } i=k
\end{array} \quad(i, k=1,2,3) .\right.
\end{gathered}
$$

Hierin ist

$$
\vec{K}_{i}^{*}=\vec{K}_{i}-\left(\operatorname{grad} \mu_{i}\right)_{T} \quad(i=1,2, \ldots N)
$$

die „Kraft“, die den Diffusionsstrom $\vec{J}_{i}$ treibt,

$$
\vec{L}_{W}=-\frac{1}{T} \operatorname{grad} T
$$

die „Kraft“, die den Wärmestrom $\vec{W}$ treibt, und

$$
L_{i k}=-\frac{1}{2}\left(\frac{\partial v_{i}}{\partial z_{k}}+\frac{\partial v_{k}}{\partial z_{i}}\right) \quad(i, k=1,2,3)
$$

die „Kraft“, die den „Impulsstrom“ $p_{i k}$ treibt. Die $\alpha_{i k}, \beta_{i}, \beta_{i}^{\prime}, \gamma$ und $a_{r s}$ sind ,phänomenologische Koeffizienten “, $\eta$ die Viskosität.

Die Gln. (100a) und (100b) sind den Ansätzen (72a) und (72b) bei polythermen Systemen analog; sie beschreiben Diffusion, Elektrizitätsleitung, Wärmeleitung und deren gegenseitige Überlagerungen (thermoelektrische Erscheinungen, Thermodiffusion usw.). Gl. (100 c) entspricht der Gl. (34) für chemische Reaktionen in homogenen Systemen. Gl. (100d) endlich ist ein hydrodynamischer Ansatz und das Analogon der „stress strain relation“, wobei der Einfachheit halber Verschwinden der „Volumenviskosität“ vorausgesetzt wurde [vgl. Meixne ${ }^{4}$ ].

Gemäß Gl. (89) ergibt sich:

$$
\sum_{i=1}^{N} a_{i k}=0, \quad \sum_{i=1}^{N} \beta_{i}=0 .
$$

Ferner gelten die Reziprozitätsbeziehungen von Onsager:

$$
\begin{gathered}
a_{i k}=a_{k i}, \\
\beta_{i}=\beta_{i}^{\prime}, \\
a_{r s}=a_{s r} .
\end{gathered}
$$

Die Gln. (105a) verknüpfen die Diffusionskoeffizienten ${ }^{43}$ der verschiedenen Stoffe miteinander, und die Gln. (105c) formulieren Bedingungen für die Geschwindigkeiten gekoppelter chemischer Reaktionen (in Gleichgewichtsnähe). Die Gln. (105b) endlich ergeben Beziehungen zwischen den Überlagerungsprozessen (Beispiel: Thermodiffusion) und den entsprechenden Umkehreffekten (Beispiel: Diffusionsthermoeffekt).

Der positiv definite Charakter der lokalen Entropieerzeugung [s. (96)] führt mit (98) und (100)—(105) $\mathrm{zu}$ folgenden Vorzeichenaussagen, wenn wir die Koeffizienten $\alpha_{N i}(i=1,2, \ldots N)$ und $\beta_{N}$ gemäß Gl. (104) eliminieren:

$$
\begin{gathered}
a_{i i}>0, \quad \gamma>0, \quad a_{i i} a_{k k}-a_{i k}^{2}>0, \\
a_{i i} \gamma-\beta_{i}^{2}>0, \quad \ldots \operatorname{det}\left(a_{i k}\right)>0, \quad \Delta>0, \\
a_{r r}>0, \quad a_{r r} a_{s s}-a_{r s}^{2}>0, \ldots \operatorname{det}\left(a_{r s}\right)>0, \\
\eta>0 .
\end{gathered}
$$

Hierin ist $\Delta$ die Determinante der Koeffizienten der quadratischen Form $T \vartheta$ :

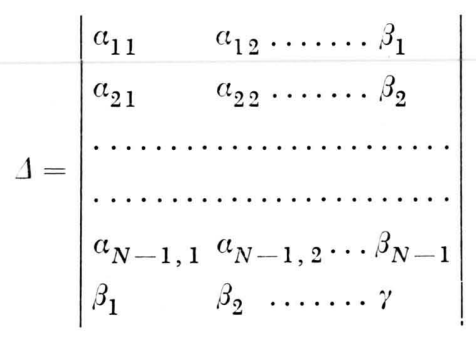

$\operatorname{det}\left(\alpha_{i k}\right)$ ist $\operatorname{der}(N-1)$-reihige Hauptminor von $\Delta$, der aus den $\alpha_{i k}$ gebildet wird.

d) Wärmeleitfähigkeit

Gl. (101) zeigt, daß bei Zugrundelegung des „reduzierten Wärmestromes“ die „Kräfte“ für die Diffusionsströme nicht mehr den Temperaturgradienten enthalten. Daraus ergeben sich — ähnlich wie bei Verwendung von $X_{Q}$ für die polythermen Systeme -

43 Genauer: die Koeffizienten für die isotherme Diffusion, d.h. die gewöhnliche Diffusion und die Druckdiffusion, bzw. für die analogen isothermen Prozesse bei der Elektrizitätsleitung. 
sowohl formale Vereinfachungen bei der Durchrechnung konkreter Fälle als auch eine physikalisch sinnvolle Festlegung des Begriffs „Wärmeleitfähigkeit“.

Wenn wir nur die $N-1$ unabhängigen Diffusionsströme betrachten, können wir mit Gl. (104) die Ansätze (100a) und (100b) auf eine Form bringen, die den direkten Vergleich mit den Ansätzen (72a) und (72b) zuläßt; die Summen sind dann nur bis $N-1$ zu erstrecken, und an die Stelle von $\vec{K}_{i}^{*} \operatorname{tritt} \vec{K}_{i}^{*}-\vec{K}_{N}{ }^{*}$.

Der Zustand „homogener Durchmischung“ ist durch die Bedingungen

$$
\vec{K}_{i}=0, \quad\left(\operatorname{grad} \mu_{i}\right)_{T}=0 \quad(i=1,2 \ldots N),
$$

der „stationäre Zustand“ (bei Fehlen chemischer Reaktionen) durch

$$
\overrightarrow{J_{i}}=0 \quad(i=1,2 \ldots N-1), \quad \vec{W}=\mathrm{const}
$$

charakterisiert, wenn der Temperaturgradient vorgegeben ist.

Aus (100 b), (101), (102) und (108) folgt für Verschwinden aller äußeren Kräfte und der Gradienten des Druckes und der Konzentrationen ${ }^{44}$ das „Fouriersche Gesetz":

$$
\vec{W}=-\lambda \operatorname{grad} T,
$$

worin

$$
\lambda \equiv \frac{\gamma}{T}
$$

die „Wärmeleitfähigkeit für homogene Durchmischung" ist.

Aus (106 a) ergibt sich:

$$
\lambda>0 \text {. }
$$

Auch hier führt nur die Verwendung des „,reduzierten Wärmestromes“ zu positivem Vorzeichen der obigen Größe.

Wir definieren $N-1$,Überführungswärmen“ $Q_{k}{ }^{*}$ durch die Gleichung [vgl. M e ix n e r ${ }^{4}$ ]

$$
\beta_{i} \equiv \sum_{k=1}^{N-1} a_{i k} Q_{k}^{*} \quad(i=1,2 \ldots N-1) .
$$

Nun verlaufen die Rechnungen formal identisch mit denen des Abschnitts $3 \mathrm{~d}$, wenn wir an Stelle von

$$
N, b_{i}, a_{i k} \cdot c, Q_{i}, X_{i}, X_{Q}, \Delta T
$$

$$
\begin{aligned}
& 44 \text { Es gilt: } \\
& \left(\operatorname{grad} \mu_{i}\right)_{T}=V_{i} \operatorname{grad} p+\sum_{k=1}^{N-1}\left(\frac{\partial \mu_{i}}{\partial x_{k}}\right)_{T, p} \operatorname{grad} x_{k},
\end{aligned}
$$

worin $V_{i}$ das partielle Volumen der Komponente $i$ ist. setzen [vgl. (75) und (107)]:

$$
N-1, \beta_{i}, a_{i k}, \gamma, Q_{i}^{*}, \overrightarrow{J_{i}}, \vec{W},-\operatorname{grad} T .
$$

Wir erhalten also für den stationären Zustand (ohne Berücksichtigung chemischer Reaktionen) wiederum eine Gleichung von der Form des „Fourierschen Gesetzes“ [vgl. (83) und (84)]:

worin

$$
\vec{W}=-\varkappa \operatorname{grad} T,
$$

$$
\varkappa \equiv \frac{1}{T}\left(\gamma-\sum_{i=1}^{N-1} \beta_{i} Q_{i}^{*}\right)
$$

die „Wärmeleitfähigkeit für den stationären $\mathrm{Zu}-$ stand". ist.

Für das Vorzeichen von $\varkappa$ und den Zusammenhang zwischen $\lambda$ und $\varkappa$ gilt [vgl. (85) und (86)]:

$$
\varkappa>0 \text {, }
$$

$$
\lambda-\varkappa=\frac{1}{T} \sum_{i=1}^{N-1} \beta_{i} Q_{i}^{*}=\frac{1}{T} \sum_{i=1}^{N-1} \sum_{k=1}^{N-1} a_{i k} Q_{i}^{*} Q_{k}^{*}>0 .
$$

Die $Q_{i}^{*}$ sind auf die Minoren der Determinante in Gl. (107) und damit auf die phänomenologischen Koeffizienten $\alpha_{i k}, \beta_{i}$ zurückführbar.

Das Beispiel der Thermodiffusion in binären Gemischen, das früher durchgerechnet wurde, bestätigt uns direkt 11, daß die mit dem Meixnerschen Wärmestrom $\vec{W}^{*}$ definierten „Wärmeleitfähigkeiten“ $\lambda^{*}$ und $\varkappa^{*}(=\varkappa)$ den Bedingungen (112) und (117) nicht genügen ${ }^{45}$. Andererseits ergeben die mit $\vec{W}$ durchgeführten Rechnungen eine explizite Form der Gl. (117) für den betrachteten Spezialfall. Es gilt nämlich, wenn wir sogleich die konventionellen Koeffizienten für die Transporterscheinungen (Diffusionskoeffizient $D$, Thermodiffusionsfaktor $\alpha$ ) einführen 12:

$$
\lambda-\varkappa=\frac{D \alpha^{2} \bar{x}_{1}^{2} \bar{x}_{2}}{T V_{m}}\left(\frac{\partial \bar{\mu}_{1}}{\partial \bar{x}_{1}}\right)_{T, p},
$$

worin $\bar{x}_{i}$ Molenbruch, $\bar{\mu}_{i}$ molares chemisches Potential der Komponente $i$ und $V_{m}$ Molvolumen der Mischung.

Am obigen Beispiel kann auch gezeigt werden ${ }^{12}$, da $\beta$ die Verwendung von $\vec{W}$ und $\lambda$ (an Stelle von $\vec{W}^{*}$ und $\lambda^{*}$ oder anderer Kombinationen von „Wärmestrom“ und „Wärmeleitfähigkeit“) eine Reihe weiterer Vereinfachungen mit sich bringt (vgl. Abschn. $3 \mathrm{~d}$ ). Insbesondere tritt in der Wärmeleitungsgleichung, die zur Auswertung von Messungen des Diffusionsthermoeffektes benutzt wird, die Größe $\lambda$ an derjenigen Stelle auf, an der ohne Thermodiffusicn usw. die „Wärmeleitfähigkeit“ schlechthin stehen würde.

45 In 11 steht $\lambda^{\prime}$ für $\lambda, \lambda$ für $\varkappa$, $\varkappa$ für $\lambda^{*}, \vec{W}$ für $\vec{W}^{*}$. 


\section{5. Üb ersicht}

Die wichtigsten Ergebnisse der hier durchgeführten allgemeinen Rechnungen können folgendermaßen zusammengefaßt werden.

Beliebige irreversible Prozesse werden in erster Näherung durch die sog. ,phänomenologischen Ansätze“, d. h. homogene lineare Beziehungen zwischen generalisierten „Strömen“ und „Kräften“, beschrieben. Legen wir die "Ströme“ durch physikalisch sinnvolle Definitionen fest, so ergeben sich die zugehörigen „Kräfte“ zwangsläufig aus der „Dissipationsfunktion", wie die Theorie im einzelnen zeigt. Betrachten wir ein System, in dem chemische Reaktionen („Ströme“: die Reaktionsgeschwindigkeiten $w_{r}$, „Kräfte“: die Affinitäten $A_{r}$ ), Stofftransport („Ströme“: $J_{i}$, zugehörige „Kräfte“: $K_{i}$ ) und Wärmeleitung („Strom“: W, zugehörige „Kraft“: $L_{u}$ ) gleichzeitig vor sich gehen, so lauten die linearen Ansätze — bei Berücksichtigung der Onsagerschen Reziprozitätsbeziehungen - in ihrer kürzesten Form:

$$
w_{r}=\sum_{s=1}^{R} a_{r s} A_{s}, \quad a_{r s}=a_{s r} \quad(r, s=1,2 \ldots R),
$$

$J_{i}=\sum_{k=1}^{n} A_{i k} K_{k}+B_{i} L_{W}, A_{i k}=A_{k i}(i, k=1,2 \ldots n)$,

$$
W=\sum_{i=1}^{n} B_{i} K_{i}+C L_{W}
$$

Hierin sind: $w_{r}$ die Geschwindigkeit der Reaktion $r$ in der betrachteten Phase bzw. (bei kontinuierlichen Systemen) im betrachteten Volumenelement (dann auf die Volumeneinheit bezogen), $\boldsymbol{J}_{i}$ der „Materiestrom“ des Stoffes $i$, d. h. die Geschwindigkeit des Massenübertritts von einer Phase zur anderen, bzw. (bei kontinuierlichen Systemen) der „Diffusionsstrom“ des Stoffes $i$, d. h. die Diffusionsstromdichte am betrachteten Ort, $W$ der „Wärmestrom“, d. h. die Wärmeübergangsgeschwindigkeit von einer Phase zur anderen bzw. (bei kontinuierlichen Systemen) die Wärmestromdichte am betrachteten Ort, $R$ die Zahl der chemischen Reaktionen, $n$ die Zahl der unabhängigen Materieströme bzw. Diffusionsströme, $a_{r s}, A_{i k}, B_{i}$ und $C$ die phänomenologischen Kòffizienten.

Setzen wir

$$
\Delta \equiv\left|\begin{array}{cccc}
A_{11} & A_{12} \ldots \ldots & B_{1} \\
A_{21} & A_{22} \ldots & \ldots & B_{2} \\
\ldots & \ldots & \ldots & \ldots \\
\ldots & \ldots & \ldots \\
B_{1} & B_{2} \ldots \ldots & \ldots & \ldots
\end{array}\right|
$$

$$
B_{i} \equiv \sum_{k=1}^{n} A_{i k} Q_{k} \quad(i=1,2 \ldots n)
$$

wodurch die $n$ „Überführungswärmen“ $Q_{k}$ definiert werden, so ergibt die Theorie folgende Aussagen:

1. Es ist möglich, durch physikalisch plausible Operationen vom gesamten „Wärmestrom“ einen Teil abzuspalten, der sich auf den Wärmetransport durch Materieströme bzw. Diffusionsströme bezieht, und so $\mathrm{zu}$ einem "reduzierten Wärmestrom“ zu gelangen.

2. Ist $W$ in Gl. (118c) der ,reduzierte Wärmestrom“, so enthalten die „Kräfte“ $K_{i}$ in Gl. (118b) und $(118 \mathrm{c})$, die zu den Materieströmen bzw. Diffusionsströmen gehören, nur noch äußere Kräfte sowie Differenzen bzw. Gradienten des Druckes und der Konzentrationen, während die Temperaturdifferenz bzw. der Temperaturgradient lediglich in der zum Wärmestrom gehörenden „Kraft“ $L_{W}$ auftritt. Für diese Wahl des „Wärmestromes“ gilt also:

a) Die Koeffizienten $A_{i k}$ beschreiben den isothermen Massentransport zwischen verschiedenen Phasen bzw. die isotherme Diffusion (oder Elektrizitätsleitung), $C$ die Wärmeleitung und die $B_{i}$ die Überlagerungsprozesse.

b) Die aus den phänomenologischen Ansätzen folgenden expliziten Gleichungen für meßbare Größen sind besonders einfach hinsichtlich der Form, in der die phänomenologischen Koeffizienten auftreten.

3. Aus dem (erweiterten) Zweiten Hauptsatz der Thermodynamik folgen die Vorzeichenaussagen:

$$
\begin{gathered}
a_{r r}>0, \quad a_{r r} a_{s s}-a_{r s}^{2}>0, \quad \ldots \operatorname{det}\left(a_{r s}\right)>0, \\
A_{i i}>0, \quad C>0, \quad A_{i i} A_{k k}-A_{i k}^{2}>0, \\
A_{i i} C-B_{i}^{2}>0 \ldots, \quad \operatorname{det}\left(A_{i k}\right)>0, \quad \Delta>0 .
\end{gathered}
$$

4. Bezeichnen wir mit $\lambda$ die „Wärmeleitfähigkeit für homogene Durchmischung " und mit $\varkappa$ die „Wärmeleitfähigkeit für den stationären Zustand (ohne chemische Reaktionen)“, so gilt:

$$
\begin{gathered}
i=\frac{C}{T}>0, \\
\%>0, \\
i-\%=\frac{1}{T} \sum_{i=1}^{n} B_{i} Q_{i}=\frac{1}{T} \sum_{i=1}^{n} \sum_{k=1}^{n} A_{i k} Q_{i} Q_{k}>0 .
\end{gathered}
$$

Die Bedingung $\lambda>0$ folgt auch unmittelbar aus (120) und (121). Bei Wahl eines anderen „Wärme- 
stromes" brauchen die Vorzeichenaussagen $\lambda>0$ und $\lambda-\varkappa>0$ nicht erfüllt zu sein. Die Notwendigkeit einer Unterscheidung zwischen $\varkappa$ und $\lambda$ ergibt sich, wohlverstanden, auch nach der vorherigen Abspaltung des „Wärmetransports durch Diffusion“; denn sie beruht auf dem Vorhandensein der Überlagerungs- prozesse (Thermoosmose, Thermodiffusion, thermoelektrische Erscheinungen usw.).

Herrn Prof. W. J o s.t danke ich dafür, daß er mir die Anregung zur Beschäftigung mit diesem Gebiet gegeben und die Durchführung der obigen Untersuchungen ermöglicht hat.

\title{
Dichroismus gereckter Folien
}

\author{
Von W. Hanle und H. Scherer \\ Aus dem Physikalischen Institut der Justus-Liebig-Hochschule Gießen \\ (Z. Naturforschg. 6 a, 437-439 [1951]; eingegangen am 28. April 1951)
}

\begin{abstract}
Es wird die anisotrope Lichtabsorption gereckter und gefärbter Kolloidfolien im Ultravioletten untersucht und gezeigt, daß durch geeignet gewählte Farbstoffe in jedem gewünschten Spektralbereich Dichroismus erhalten werden kann.
\end{abstract}

$\mathrm{W}_{\text {wat }}$ erden durchsichtige Folien z. B. aus Cellulosehydrat gereckt und dann durch Eintauchen in eine Farbstofflösung angefärbt, so zeigen sie häufig Dichroismus. Das parallel zur Reckrichtung schwingende Licht hat eine andere Absorption, mitunter auch ein anderes Absorptionsspektrum, als das senkrecht zur Reckrichtung schwingende Licht. Die Stärke des Dichroismus ändert sich von Fall zu Fall. Bislang wurden solche Untersuchungen nur im sichtbaren Spektralbereich ausgeführt. Wir haben uns die Aufgabe gestellt, sie auf das Ultraviolett auszudehnen, und zwar aus zwei Gesichtspunkten: zum einen war erst durch die Ausdehnung des Spektralbereichs die ganze Fülle an Erscheinungen zu übersehen und zum anderen wollten wir untersuchen, wie weit mit dieser Methode die Herstellung von Polarisationsfiltern für ultraviolettes Licht möglich ist.

Die Untersuchungen wurden zum Teil photographisch, zum Teil lichtelektrisch ausgeführt. Bei der photographischen Methode wurde ein gleichmäßig beleuchteter Spalt unter Zwischenschaltung eines Kalkspates so auf den Spalt eines Quarzspektrographen abgebildet, daß die Bilder des horizontal und vertikal schwingenden Lichtes übereinander lagen. Man erhält dann entsprechend zwei übereinander liegende Spektren, in denen das Licht horizontal und vertikal polarisiert ist. Bringt man die zu untersuchende Folie so vor den Spalt des Spektrographen, daß ihre Reckrichtung senkrecht oder parallel liegt, so erhält man die Absorptionsspektren in den beiden Schwingungsebenen parallel und senkrecht zur Reck- richtung. Ihr Vergleich gibt die Stärke der Polarisation in den einzelnen Spektralbereichen. Die Folien, welche als Polarisationsfilter gut geeignet und für weitere Untersuchungen interessant schienen, wurden lichtelektrisch ausgemessen. Hierbei diente zur spektralen Zerlegung des Lichtes ein Quarzdoppelmonochromator. Das aus ihm austretende monochromatische Licht wurde mittels eines Kalkspates in zwei senkrecht zueinander polarisierte Teilbündel aufgespalten, von denen jeweils der eine durch eine verschiebbare Blende ausgesondert wurde. Die zu untersuchenden Folien wurden wie in der photographischen Anordnung in den Strahlengang gebracht. Die Intensitäten wurden mit Photozelle und Elektrometerverstärker bestimmt.

Eine große Zahl von Farbstoffen wurde untersucht. In Tab. 1 sind diejenigen aufgeführt, welche Dichroismus aufweisen. Abb. $1^{*}$ zeigt einige Beispiele der Spektralaufnahmen. Man kann praktisch jede Art der Polarisation erzielen. Es gibt Farbstoffe, welche über den ganzen untersuchten Spektralbereich vom Roten bis $2500 \AA$ gut polarisieren. Andere polarisieren nur in einem gewissen Spektralbereich, z. B. Thiazolgelb im langwelligen und kurzwelligen UV, im Sichtbaren auch noch im Violetten, dagegen nicht mehr im Grünen. - Diazurin B polarisiert gut im Sichtbaren, dagegen nicht im UV. - Methylenblau BGx polarisiert gut im kurzwelligen UV, nicht im langwelligen und absorbiert vollständig vom Gelben ab. Diazoblauschwarz RS polarisiert gut im Sicht-

* Abb. 1, s. Tafel S. 440 a. 